\title{
Hydrodynamical Models of Superfluid Turbulence
}

\author{
D. Jou ${ }^{1}$, M.S. Mongiovì ${ }^{2}$, M. Sciacca ${ }^{2}$, L. Ardizzone ${ }^{2}$ and G. Gaeta ${ }^{2}$ \\ ${ }^{1}$ Departament de Física, Universitat Autònoma de Barcelona, Bellaterra, Catalonia \\ ${ }^{2}$ Dipartimento di Metodi e Modelli Matematici, Università di Palermo, Palermo \\ ${ }^{1}$ Spain \\ ${ }^{2}$ Italy
}

\section{Introduction}

Turbulence is almost the rule in the flow of classical fluids. It is a complex nonlinear phenomenon for which the development of a satisfactory theoretical framework is still incomplete. Turbulence is often found in the flow of quantum fluids, especially superfluid Helium 4, known as liquid helium II (Donnelly, 1991), (Nemirovskii \& Fiszdon, 1995), (Barenghi et al., 2001), (Vinen \& Niemela, 2002).

In recent years there has been growing interest in superfluid turbulence, because of its unique quantum peculiarities and of its similarity with classical turbulence to which it provides a wide range of new experimental possibilities at very high Reynolds numbers (Vinen, 2000), (Barenghi, 1999), and because of their influence in some practical applications, as in refrigeration by means of superfluid helium. We will consider here the turbulence in superfluid ${ }^{4} \mathrm{He}$, for which many detailed experimental techniques have been developed.

The behavior of liquid helium, below the lambda point $\left(T_{c} \simeq 2.17 \mathrm{~K}\right)$, is very different from that of ordinary fluids. One example of non-classical behavior is the possibility to propagate the second sound, a wave motion in which temperature and entropy oscillate. A second example of non-classical behavior is heat transfer in counterflow experiments. Using an ordinary fluid (such as helium I), a temperature gradient can be measured along the channel, which indicates the existence of a finite thermal conductivity. If helium II is used, and the heat flux inside the channel is not too high, the temperature gradient is so small that it cannot be measured, so indicating that the liquid has an extremely high thermal conductivity (three million times larger than that of helium I). This is confirmed by the fact that helium II is unable to boil. This effect explains the remarkable ability of helium II to remove heat and makes it important in engineering applications.

The most known phenomenological model, accounting for many of the properties of $\mathrm{He}$ II, given by Tisza and Landau (Tisza, 1938), (Landau, 1941) is called the two-fluid model. The basic assumption is that the liquid behaves as a mixture of two fluids: the normal component with density $\rho_{n}$ and velocity $\mathbf{v}_{n}$, and the superfluid component with density $\rho_{s}$ and velocity $\mathbf{v}_{s}$, with total mass density $\rho$ and barycentric velocity $\mathbf{v}$ defined by $\rho=\rho_{s}+\rho_{n}$ and $\rho \mathbf{v}=\rho_{s} \mathbf{v}_{S}+\rho_{n} \mathbf{v}_{n}$. The second component is related to the quantum coherent ground state and it is an ideal fluid, which does not experience dissipation neither carries entropy. The superfluid component, which is absent above the lambda transition temperature, was originally considered to be composed by particles in the Bose-Einstein state and is an ideal 
fluid, and the normal component by particles in the excited state (phonons and rotons) and is a classical Navier-Stokes viscous fluid.

The two-fluid model explains the experiment described above in the following way: in the absence of mass flux $\left(\rho_{n} \mathbf{v}_{n}+\rho_{s} \mathbf{v}_{S}=0\right.$ and $\mathbf{v}_{n}$ and $\mathbf{v}_{S}$ averaged on a small mesoscopic volume $\Lambda$ ), in helium II the heat is carried toward the bath by the normal fluid only, and $\mathbf{q}=\rho s T \mathbf{v}_{n}$ where $s$ is the entropy per unit mass and $T$ the temperature. Being the net mass flux zero, there is superfluid motion toward the heater $\left(\mathbf{v}_{s}=-\rho_{n} \mathbf{v}_{n} / \rho_{s}\right)$, hence there is a net internal counterflow $\mathbf{V}_{n s}=\mathbf{v}_{n}-\mathbf{v}_{s}=\mathbf{q} /\left(\rho_{s} s T\right)$ which is proportional to the applied heat flux $\mathbf{q}$.

An alternative model of superfluid helium is the one-fluid model (Lebon \& Jou, 1979), (Mongiovì, 1993), (Mongiovì, 2001) based on extended thermodynamics (Müller \& Ruggeri, 1998), (Jou et al., 2001), (Lebon et al., 2008). Extended Thermodynamics (E.T.) is a thermodynamic formalism proposed in the last decades, which offers a natural framework for the macroscopic description of liquid helium II. The basic idea underlying E.T. is to consider the physical fluxes as independent variables. In previous papers, the E.T. has been applied to formulate a non-standard one-fluid model of liquid helium II, for laminar flows. This model is recalled in Section 2, in the absence of vortices (laminar flow) and in Section 3 both in rotating containers and in counterflow situations.

Quantum turbulence is described as a chaotic tangle of quantized vortices of equal circulation

$$
\kappa=\oint \mathbf{u}_{s} \cdot d l
$$

( $\mathbf{u}_{s}$ microscopic velocity of the superfluid component) called quantum of vorticity and results $\kappa=h / m_{4}$, with $h$ the Planck constant, and $m_{4}$ the mass of ${ }^{4} \mathrm{He}$ atom: $\kappa \simeq 9.9710^{-4} \mathrm{~cm}^{2} / \mathrm{s}$. Since the vorticity is quantized, the increase of turbulence is manifested as an increase of the total length of the vortex lines, rather than with a faster spinning of the vortices. Thus, the dynamics of the vortex length is a central aspect of quantum turbulence.

A preliminary study of these interesting phenomena was made in (Jou et al., 2002), where the presence of vortices was modeled through a pressure tensor $\mathbf{P}_{\omega}$ for which a constitutive relation was written. In homogeneous situations, the vortex tangle is described by introducing a scalar quantity $L$, the average vortex line length per unit volume (briefly called vortex line density). The evolution equation for $L$ in counterflow superfluid turbulence has been formulated by Vinen (Vinen, 1958), (Donnelly, 1991), (Barenghi et al., 2001)

$$
\frac{d L}{d t}=\alpha_{v} V_{n s} L^{3 / 2}-\beta_{v} \kappa L^{2},
$$

with $V_{n s}$ the modulus of the counterflow velocity $\mathbf{V}_{n s}=\mathbf{v}_{n}-\mathbf{v}_{s}$, which is proportional to the heat flux $\mathbf{q}$, and $\alpha_{v}$ and $\beta_{v}$ dimensionless parameters. This equation assumes homogeneous turbulence, i.e. that the value of $L$ is the same everywhere in the system. In fact, homogeneity may be expected if the average distance between the vortex filaments, of the order of $L^{-1 / 2}$, is much smaller than the size of the system.

Recent experiments show the formation of a new type of superfluid turbulence, which has some analogies with classical one, as for instance using towed or oscillating grids, or stirring liquid helium by means of propellers. In this situation, which has been called co-flow, both components, normal and superfluid, flow along the same direction. To describe these experiments it is necessary to build up a hydrodynamic model of quantum turbulence, in which the interactions between both fields can be studied and the role of inhomogeneities is explicitly taken into account. 
Our aim in this review is to show hydrodynamical models for turbulent superfluids, both in linear and in non linear regimes. To this purpose, in Section 4 we will choose as fundamental fields the density $\rho$, the velocity $\mathbf{v}$, the internal energy density $E$, in addition to the heat flux q, and the averaged vortex line density L (Mongiovì \& Jou, 2007), (Ardizzone \& Gaeta, 2009). We will write general balance equations for the basic variables and we will determine the constitutive equations for the fluxes; the nonlinear relations which constrain the constitutive quantities will be deduced from the second law of thermodynamics, using the Liu method of Lagrange multipliers (Liu, 1972). The physical meaning of the Lagrange multipliers both near and far from equilibrium will be also investigated. Under the hypothesis of homogeneity in the vortex tangle, the propagation of second sound in counterflow is studied, with the aim to determine the influence of the vortex tangle on the velocity and attenuation of this wave. In this model the diffusion flux of vortices $\mathbf{J}^{L}$ is considered as a dependent variable, collinear with the heat flux q. But, in general, this feature is not strictly verified because the vortices move with a velocity $\mathbf{v}_{L}$, which is not collinear with the counterflow velocity. For this reason, a more detailed model of superfluid turbulence would be necessary, by choosing as fundamental fields, in addition to the fields previously used, also the velocity of the vortex line $\mathbf{v}_{L}$. In Section 5 we aim to study the interaction between second sound and vortex density wave, a model which choose as field variables, the internal energy density $E$, the line density $L$, and the vortex line velocity $\mathbf{v}_{L}$ (Sciacca et al, 2008).

The paper is the first general review of the hydrodynamical models of superfluid turbulence inferred using the procedures of E.T. Furthermore, the text is not exclusively a review of already published results, but it contains some new interpretations and proposals which are formulated in it for the first time.

\section{The one-fluid model of liquid helium II derived by extended thermodynamics}

Extended Thermodynamics (E.T.) is a macroscopic theory of non-equilibrium processes, which has been formulated in various ways in the last decades (Müller \& Ruggeri, 1998), (Jou et al., 2001), (Lebon et al., 2008). The main difference between the ordinary thermodynamics and the E.T. is that the latter uses dissipative fluxes, besides the traditional variables, as independent fields. As a consequence, the assumption of local equilibrium is abandoned in such a theory. In the study of non equilibrium thermodynamic processes, an extended approach is required when one is interested in sufficiently rapid phenomena, or else when the relaxation times of the fluxes are long; in such cases, a constitutive description of these fluxes in terms of the traditional field variables is impossible, so that they must be treated as independent fields of the thermodynamic process.

From a macroscopic point of view, an extended approach to thermodynamics is required in helium II because the relaxation time of heat flux is comparable with the evolution times of the other variables; this is confirmed by the fact that the thermal conductivity of helium II cannot be measured. As a consequence, this field cannot be expressed by means of a constitutive equation as a dependent variable, but an evolution equation for it must be formulated.

From a microscopic point of view, E.T. offers a natural framework for the (macroscopic) description of liquid helium II: indeed, as in low temperature crystals, using E.T., the dynamics of the relative motion of the excitations is well described by the dynamics of the heat flux.

The conceptual advantage of the one-fluid model is that, in fact, from the purely macroscopic point of view one sees only a single fluid, rather than two physically different fluids. Indeed the variables $\mathbf{v}$ and $\mathbf{q}$ used in E.T. are directly measurable, whereas the variables $\mathbf{v}_{n}$ and $\mathbf{v}_{S}$, 
are only indirectly measured, usually from the measurements of $\mathbf{q}$ and $\mathbf{v}$. The internal degree of freedom arising from the relative motion of the two fluids is here taken into account by the heat flux, whose relaxation time is very long. However, the two-fluid model provides a very appealing image of the microscopic helium behavior, and therefore is the most widely known.

\subsection{Laminar flows}

A non standard one-fluid model of liquid helium II deduced by E.T. was formulated in (Mongiovì, 1991). The model chooses as fundamental fields the mass density $\rho$, the velocity $\mathbf{v}$, the absolute temperature $T$ and the heat flux density $\mathbf{q}$. Neglecting, at moment, dissipative phenomena (mechanical and thermal), the linearized evolution equations for these fields are:

$$
\left\{\begin{array}{l}
\dot{\rho}+\rho \nabla \cdot \mathbf{v}=0, \\
\rho \dot{\mathbf{v}}+\nabla p^{*}=0, \\
\rho \dot{\epsilon}+\nabla \cdot \mathbf{q}+p \nabla \cdot \mathbf{v}=0, \\
\dot{\mathbf{q}}+\zeta \nabla T=0 .
\end{array}\right.
$$

In these equations, the quantity $\epsilon$ is the specific internal energy per unit mass, $p$ the thermostatic pressure, and $\zeta=\lambda_{1} / \tau$, being $\tau$ the relaxation time of the heat flux and $\lambda_{1}$ the thermal conductivity. As it will be shown, coefficient $\zeta$ characterizes the second sound velocity, and therefore it is a measurable quantity. Upper dot denotes the material time derivative.

Equations (3) describe the propagation in liquid helium II of two waves, whose speeds $w$ are the solutions of the following characteristic equation:

$$
\left(w^{2}-V_{1}^{2}\right)\left(w^{2}-V_{2}^{2}\right)-W_{1} W_{2} u^{2}=0,
$$

where

$$
V_{1}^{2}=p_{\rho}, \quad V_{2}^{2}=\frac{\zeta}{\rho c_{V}}, \quad W_{1}=\frac{p_{T}}{\rho}, \quad W_{2}=\frac{T p_{T}}{\rho c_{V}},
$$

and with $c_{V}=\partial \epsilon / \partial T$ the constant volume specific heat and $p_{T}=\partial p / \partial T$ and $p_{\rho}=\partial p / \partial \rho$. Neglecting thermal expansion $\left(W_{1}=0, W_{2}=0\right)$ equation (4) admits the solutions $w_{1,2}= \pm V_{1}$ and $w_{3,4}= \pm V_{2}$, corresponding to the two sounds typical of helium II: $w= \pm V_{1}$ implies vibration of only density and velocity; while $w= \pm V_{2}$ implies vibration of only temperature and heat flux. This agrees with the experimental observations. The coefficient $\zeta$ can be determined by the second equation in 5 , once the expression of the second sound velocity is known.

Finally, we observe that the Gibbs equation for helium II can be written as

$$
T d s=d \epsilon-\frac{p}{\rho^{2}} d \rho-\frac{1}{\rho \zeta T} \mathbf{q} \cdot d \mathbf{q},
$$

where $s$ is the specific entropy.

\subsection{The viscous pressure tensor}

It is experimentally known that dissipative effects both of mechanical and thermal origin are present in the propagation of the two sounds in liquid helium II, also in the absence of 
vortices. To take into account of these effects, a symmetric dissipative pressure tensor $\mathbf{P}_{K}$ must be introduced:

$$
\left[\mathbf{P}_{K}\right]_{i k}=p_{<i k>}+p_{V} \delta_{i k} .
$$

In (Mongiovì, 1993) for the two fields $p_{<i j>}$ and $p_{V}$, respectively deviator and trace of the stress tensor, the following constitutive relations were determined:

$$
\begin{gathered}
p_{V}=-\lambda_{0} \frac{\partial v_{j}}{\partial x_{j}}+\beta^{\prime} T \lambda_{0} \frac{\partial q_{j}}{\partial x_{j}}, \\
p_{<i k>}=-2 \lambda_{2} \frac{\partial v_{<i}}{\partial x_{k>}}+2 \beta T \lambda_{2} \frac{\partial q_{<i}}{\partial x_{k>}} .
\end{gathered}
$$

In these equations $\lambda_{0}$ and $\lambda_{2}$ are the bulk and the shear viscosity, while $\beta$ and $\beta^{\prime}$ are coefficients appearing in the general expression of the entropy flux in E.T. and take into account of the dissipation of thermal origin.

Equations (8)-(9) contain, in addition to terms proportional to the gradient of velocity (the classical viscous terms), terms depending on the gradient of the heat flux (which take into account of the dissipation of thermal origin). The first terms in (8)-(9) allow us to explain the attenuation of the first sound, the latter the attenuation of the second sound.

In the presence of dissipative phenomena, the field equations (3) are modified in:

$$
\left\{\begin{array}{l}
\dot{\rho}+\rho \nabla \cdot \mathbf{v}=0 \\
\dot{\mathbf{v}}+\frac{1}{\rho} \nabla p+\frac{1}{\rho} \nabla p_{V}+\frac{1}{\rho} \nabla p_{<j i>}=0 \\
\dot{T}+\frac{T p_{T}}{\rho c_{V}} \nabla \cdot \mathbf{v}+\frac{1}{\rho c_{V}} \nabla \cdot \mathbf{q}=0 \\
\dot{\mathbf{q}}+\zeta \nabla T-\beta^{\prime} T^{2} \zeta \nabla p_{V}+\beta T^{2} \zeta \nabla p_{<j i>}=0 .
\end{array}\right.
$$

The propagation of small amplitude waves was studied in (Mongiovì, 1993). Supposing zero thermal expansion under the hypothesis of small dissipative losses (viscous and thermal) approximation, one sees that in helium II two waves propagate (the first and the second sound), whose velocities are identical to that found in the absence of dissipation, and the attenuation coefficients are found to be:

$$
k_{s}^{(1)}=\frac{\omega^{2}}{2 \rho w_{1}^{3}}\left(\lambda_{0}+\frac{4}{3} \lambda_{2}\right), \quad k_{s}^{(2)}=\frac{\omega^{2} T^{3} \zeta}{2 w_{2}^{3}}\left(\lambda_{0} \beta^{\prime 2}+\frac{4}{3} \lambda_{2} \beta^{2}\right) .
$$

\subsection{Comparison with the two-fluid model}

Comparing these results with the results of the two-fluid model (Mongiovì, 1993), we observe that the expression of the attenuation coefficient $k_{s}^{(1)}$ of the first sound is identical to the one inferred by Landau and Khalatnikov, using the two-fluid model (Khalatnikov, 1965). The attenuation coefficient of the second sound appears different from the one obtained in (Khalatnikov, 1965). However, it contains a term proportional to the square of the frequency $\omega$, in agreement with the experimental results.

The main difference between the results of the one-fluid theory and the two-fluid model is that, while in the latter the thermal dissipation (needed to explain the attenuation of the 
second sound) is due to a dissipative term of a Fourier type, in the extended model it is a consequence of terms dependent on the gradient of the heat flux $q_{i}$ (which are present in the expressions of the trace and the deviator of non equilibrium stress, besides the traditional viscous terms).

\section{Vortices in liquid helium II}

From the historical and conceptual perspectives, the first observations of the peculiar aspects of rotation in superfluids arose in the late 1950's, when it was realized that vorticity may appear inside superfluids and that it is quantized, its quantum $\kappa$ being $\kappa=h / m_{4}$, with $h$ the Planck constant and $m_{4}$ the mass of the particles. According to the two-fluid model of Tisza and Landau (Tisza, 1938), (Landau, 1941), the superfluid component cannot participate to a rigid rotation, owing to its irrotationality. Consequently, owing to the temperature dependence of the normal component fraction, different forms of the liquid free surface should be observed at different temperatures. In order to check this prediction, Osborne (Osborne, 1950) put in rotation a cylindrical vessel containing helium II, but no dependence of the form of the free surface of temperature was observed. Feynman (Feynman, 1955) gave an explanation of the rigid rotation of helium II without renouncing to the hypothesis of the irrotationality of the velocity of the superfluid. Following the suggestion of the quantization of circulation by Onsager (Onsager, 1949), he supposed that the superfluid component, although irrotational at the microscopic level, creates quantized vortices at an intermediate level; these vortices yield a non-zero value for the curl of the macroscopic velocity of the superfluid component.

Another interesting experiment was performed by Hall and Vinen (Hall \& Vinen,, 1956), (Hall \& Vinen,, 1956) about propagation of second sound in rotating systems. A resonant cavity is placed inside a vessel containing He II, and the whole setting rotates at constant angular velocity $\Omega$. When the second sound propagates at right angles with respect to the rotation axis, it suffers an extra attenuation compared to a non-rotating vessel of an amount proportional to the angular velocity. On the other hand, a negligible attenuation of the second sound is found when the direction of propagation is parallel to the axis of rotation. The large increase of the attenuation observed by Hall and Vinen when the liquid is rotated can be explained by the mutual friction, which finds its origin in the interaction between the flow of excitations (phonons and rotons) and the array of straight quantized vortex filaments in helium II. Indeed, such vortices have been directly observed and quantitatively studied.

In fact, vortices are always characterized by the same quantum of vorticity, in such a way that for higher rotation rates the total length of the vortices increases. The vortices are seen to form a regular array of almost parallel lines. This has strong similarities with electrical current vortex lines appearing in superconductors submitted to a high enough external magnetic field. In fact, this analogy has fostered the interest in vortices in superfluids, which allow one to get a better understanding of the practically relevant vortices in superconductors (Fazio \& van der Zant, 2001).

The situation we have just mentioned would scarcely be recognized as "turbulence", because its highly ordered character seems very far from the geometrical complexities of usual turbulence. In fact, it only shares with it the relevance of vorticity, but it is useful to refer to it, as it provides a specially clear understanding of the quantization of vorticity.

The interest in truly turbulent situations was aroused in the 1960's in counterflow experiments (Vinen, 1957), (Vinen, 1958). In these experiments a random array of vortex filaments appears, which produces a damping force: the mutual friction force. The measurements of vortex 
lines are described as giving a macroscopic average of the vortex line density $L$. There are essentially two methods to measure $L$ in superfluid ${ }^{4} \mathrm{He}$ : observations of temperature gradients in the channel and of changes in the attenuation of the second-sound waves (Donnelly, 1991), (Barenghi et al., 2001).

In the present section, our attention is focused on the study of the action of vortices on second sound propagation in liquid helium II. This will be achieved by using the one-fluid model of liquid helium II derived in the framework of E.T., modified in order to take into account of the presence of vortices.

\subsection{The vorticity tensor}

To take into account the dissipation due to vortices, a dissipative pressure tensor $\mathbf{P}_{\omega}$ can be introduced in equations (3) (Jou et al., 2002)

$$
\mathbf{P}=\mathbf{P}_{K}+\mathbf{P}_{\omega}
$$

where $\mathbf{P}_{K}$ designates the kinetic pressure tensor introduced in the previous section (equation (7)). In contrast with $\mathbf{P}_{K}$ (a symmetric tensor), $\mathbf{P}_{\omega}$ is in general nonsymmetric. The decomposition (12) is analogous to the one performed in real gases and in polymer solutions, where particle interaction or conformational contributions are respectively included as additional terms in the pressure tensor (Jou et al., 2001).

As in the description of the one-fluid model of liquid helium II made in Section 2 (see also (Mongiovì, 1991), (Mongiovì, 1993)), the relative motion of the excitations may still be described by the dynamics of the heat flux, but now the presence of the vortices modifies the evolution equation for heat flux. For the moment, we will restrict our attention to stationary situations, in which the vortex filaments are supposed fixed, and we focus our attention on their action on the second sound propagation. In other terms, in this section, we do not assume that $\mathbf{P}_{\omega}$ is itself governed by an evolution equation, but that it is given by a constitutive relation. Furthermore, we neglect $\mathbf{P}_{K}$ as compared to $\mathbf{P}_{\omega}$, because the mutual friction effects are much greater than bulk and shear forces acting inside the superfluid.

Let us now reformulate the evolution equation for the heat flux $\mathbf{q}$. The experimental data show that the extra attenuation due to the vortices is independent of the frequency. Therefore, a rather natural generalization of the last equation in system (3) for the time evolution of the heat flux $\mathbf{q}$ is the following:

$$
\dot{\mathbf{q}}+2 \Omega \times \mathbf{q}+\zeta \nabla T=-\mathbf{P}_{\omega} \cdot \mathbf{q} .
$$

This relation is written in a noninertial system, rotating at uniform velocity $\Omega$; the influence of the vortices on the dynamics of the heat flux is modeled by the last term in the r.h.s. of (13). In this equation all the non linear terms have been neglected, with the exception of the production term $\vec{\sigma}_{q}=-\mathbf{P}_{\omega} \cdot \mathbf{q}$, which takes into account the interaction between vortex lines and heat flux.

To close the set of equations, we need a constitutive relation for the tensor $\mathbf{P}_{\omega}$. The presence of quantized vortices leads to a interaction force with the excitations in the superfluid known as mutual friction. From a microscopic point of view, the major source of mutual friction results from the collision of rotons with the cores of vortex lines: the quasiparticles scatter off the vortex filaments and transfer momentum to them. The collision cross-section is clearly a strong function of the direction of the roton drift velocity relative to the vortex line: it is a maximum when the roton is travelling perpendicular to this line and a minimum (in fact zero) 
when the roton moves parallel to the line. The microscopic mechanism is the same in rotating helium II and in superfluid turbulence.

We are therefore led to take:

$$
\mathbf{P}_{\omega}=\lambda<\omega><\mathbf{U}-\mathbf{s}^{\prime} \otimes \mathbf{s}^{\prime}>+\lambda^{\prime}<\omega><\mathbf{W} \cdot \mathbf{s}^{\prime}>,
$$

where brackets denote (spatial and temporal) macroscopic averages. The unspecified quantities introduced in (14) are the following: $\vec{\omega}$ is the microscopic vorticity vector, $\omega=|\vec{\omega}|$; $\lambda=\lambda(\rho, T)$ and $\lambda^{\prime}=\lambda^{\prime}(\rho, T)$ are coefficients relating the internal energy of the liquid to the microscopic vorticity (Khalatnikov, 1965), $\mathbf{s}^{\prime}$ is a unit vector tangent to the vortices, $U$ the unit second order tensor and $\mathbf{W}$ the Ricci tensor, an antisymmetric third order tensor such that $\mathbf{W} \cdot \mathbf{s}^{\prime} \cdot \mathbf{q}=-\mathbf{s}^{\prime} \times \mathbf{q}$. Finally, the quantity $\langle\omega\rangle$ depends on the average vortex line length per unit volume $L$. Neglecting the bulk and shear viscosity and under the hypothesis of small thermal dilation (which in helium II are very small), the linearized system of field equations for liquid helium II, in a non inertial frame and in absence of external force, is (Jou et al., 2002):

$$
\left\{\begin{array}{l}
\frac{\partial \rho}{\partial t}+\rho \frac{\partial v_{j}}{\partial x_{j}}=0, \\
\rho \frac{\partial v_{i}}{\partial t}+\frac{\partial p}{\partial x_{i}}+\mathbf{i}_{i}^{0}+2 \rho(\boldsymbol{\Omega} \wedge \mathbf{v})_{i}=0, \\
\frac{\partial T}{\partial t}+\frac{1}{\rho c_{V}} \frac{\partial q_{j}}{\partial x_{j}}=0, \\
\frac{\partial q_{i}}{\partial t}+\zeta \frac{\partial T}{\partial x_{i}}+2(\boldsymbol{\Omega} \wedge \mathbf{q})_{i}=\left(\vec{\sigma}_{q}\right)_{i}=-\left(\mathbf{P}_{\omega} \cdot \mathbf{q}\right)_{i},
\end{array}\right.
$$

where $\mathbf{i}^{0}+2 \rho(\boldsymbol{\Omega} \wedge \mathbf{v})_{i}$ stands for the inertial force.

In this section we consider the three most characteristic situations: the wave propagation in a rotating frame, the wave propagation in a cylindrical tube in presence of stationary thermal counterflow (no mass flux), and the wave propagation in the combined situation of rotation and thermal counterflow.

\subsection{Rotating frame}

Rotating helium II is characterized by straight vortex filaments, parallel to the rotation axis, when the angular velocity exceeds a critical value. The amount of these vortices is proportional to the absolute value of the angular velocity $\Omega$ of the cylinder by the Feynman's rule: $L_{R}=2|\Omega| / \kappa$. Therefore

$$
<\omega>=\kappa L=2|\Omega| .
$$

In this situation the averaged unit vector tangent to the vortices is $\left\langle\mathbf{s}^{\prime}\right\rangle=\Omega / \Omega$.

But, the state with all the vortex lines parallel to the rotation axis will not be reached, because the vortex lines will always exhibit minuscule deviations with respect to the straight line, and such deviations produce a mutual friction force parallel to the rotation axis. Indeed, in an another experiment (Snyder \& Putney, 1966) the component of the mutual friction along the rotational axis was studied, and their result shows that this component is very small compared with the orthogonal components but not exactly zero. In this subsection, in order to include the axial component of the mutual friction force, the following more general expression for vorticity tensor $\mathbf{P}_{\omega}$ is used: 


$$
\mathbf{P}_{\omega}^{R}=\frac{1}{2} \kappa L_{R}\left[\left(B-B^{\prime \prime}\right)(\mathbf{U}-\hat{\mathbf{\Omega}} \otimes \hat{\mathbf{\Omega}})+B^{\prime} \mathbf{W} \cdot \hat{\mathbf{\Omega}}+2 B^{\prime \prime} \hat{\mathbf{\Omega}} \otimes \hat{\mathbf{\Omega}}\right],
$$

where $B$ and $B^{\prime}$ are the Hall-Vinen coefficients (Hall \& Vinen,, 1956) describing the orthogonal dissipative and non dissipative contributions while $B^{\prime \prime}$ is the friction coefficient along the rotational axis. The production term in (15d) can be expressed as (Donnelly, 1991), (Jou \& Mongiovì, 2005), (Jou \& Mongiovì, 2006):

$$
\vec{\sigma}_{q}^{R}=\frac{1}{2} \kappa L_{R}\left[\left(B-B^{\prime \prime}\right) \hat{\mathbf{\Omega}} \wedge(\hat{\mathbf{\Omega}} \wedge \mathbf{q})+B^{\prime} \hat{\mathbf{\Omega}} \wedge \mathbf{q}-2 B^{\prime \prime} \hat{\mathbf{\Omega}} \otimes \hat{\mathbf{\Omega}} \cdot \mathbf{q}\right]
$$

Assuming the rotation axis as first axis, the vorticity tensor (17) can be written as:

$$
\mathbf{P}_{\omega}=\frac{1}{2} B \kappa L\left\{\left(\begin{array}{ccc}
2 b & 0 & 0 \\
0 & 1-b & 0 \\
0 & 0 & 1-b
\end{array}\right)+\left(\begin{array}{ccc}
0 & 0 & 0 \\
0 & 0 & c \\
0 & -c & 0
\end{array}\right)\right\} .
$$

where we have put $b=B^{\prime \prime} / B$ and $c=B^{\prime} / B$. Comparing (19) with (14): if $B^{\prime \prime}=0$ then $B=2 \lambda, B^{\prime}=2 \lambda^{\prime},<\left(s_{x_{1}}^{\prime}\right)^{2}>=1$ and $<\left(s_{x_{2}}^{\prime}\right)^{2}>=<\left(s_{x_{3}}^{\prime}\right)^{2}>=0$; if $B^{\prime \prime} \neq 0$ then the previous identification is not possible but it results $\left.<\left(s_{x_{1}}^{\prime}\right)^{2}\right\rangle=1-2 B^{\prime \prime} / B$ and $\left.\left.<\left(s_{x_{2}}^{\prime}\right)^{2}\right\rangle=<\left(s_{x_{3}}^{\prime}\right)^{2}\right\rangle=$ $2 B^{\prime \prime} / B$.

\subsubsection{Wave propagation in a rotating frame}

In the following we assume that $\Omega$ is small, so that the term $\mathbf{i}_{0}$ in $(15 \mathrm{~b})$ can be neglected. Substituting the expression (18) into the system (15) and choosing $\Omega=(\Omega, 0,0)$, the system assumes the following form:

$$
\left\{\begin{array}{l}
\frac{\partial \rho}{\partial t}+\rho \frac{\partial v_{j}}{\partial x_{j}}=0 \\
\rho \frac{\partial v_{i}}{\partial t}+\frac{\partial p}{\partial x_{i}}+2 \rho \Omega v_{j} W_{1 j i}=0 \\
\frac{\partial T}{\partial t}+\frac{1}{\rho c_{V}} \frac{\partial q_{j}}{\partial x_{j}}=0 \\
\frac{\partial q_{i}}{\partial t}+\zeta \frac{\partial T}{\partial x_{i}}+\left(2 \Omega-\frac{1}{2} B^{\prime} \kappa L_{R}\right) q_{j} W_{1 j i}=\frac{1}{2} \kappa L_{R}\left[\left(B-B^{\prime \prime}\right)\left(-q_{i}+q_{1} \delta_{i 1}\right)-2 B^{\prime \prime} q_{1} \delta_{i 1}\right]
\end{array}\right.
$$

where $\delta_{i j}$ is the unit tensor and $W_{k j i}$ the Ricci tensor.

It is easily observed that a stationary solution of this system is:

$$
\rho=\rho_{0}, \quad \mathbf{v}=\mathbf{0}, \quad T=T_{0}, \quad \mathbf{q}=\mathbf{0} .
$$

In order to study the propagation of plane harmonic waves of small amplitude (Whitham, 1974), we linearize system (20) in terms of the fields $\Gamma=\left(\rho, v_{i}, T, q_{i}\right)$, and we look for solutions of the form:

$$
\Gamma=\Gamma_{0}+\tilde{\Gamma} e^{i\left(K n_{j} x_{j}-\omega t\right)}
$$


where $\Gamma_{0}=\left(\rho_{0}, 0, T_{0}, 0\right)$ denotes the unperturbed state, $\tilde{\Gamma}=\left(\tilde{\rho}, \tilde{v}_{i}, \tilde{T}, \tilde{q}_{i}\right)$ are small amplitudes whose products can be neglected, $K=k_{r}+i k_{s}$ is the wavenumber, $\omega=\omega_{r}+i \omega_{s}$ the frequency and $\mathbf{n}=\left(n_{i}\right)$ the unit vector orthogonal to the wave front. For the sake of simplicity, the subscript 0 , which denotes quantities referring to the unperturbed state $\Gamma_{0}$, will be dropped out.

First case: $\mathbf{n}$ parallel to $\mathbf{\Omega}$.

Assuming that the unit vector $\mathbf{n}$ orthogonal to the wave front is parallel to the rotating axis $\left(x_{1}\right.$-axis), it follows that longitudinal and transversal modes evolve independently. The study of the longitudinal modes $\left(\tilde{\rho}, \tilde{v}_{1}, \tilde{T}\right.$ and $\left.\tilde{q}_{1}\right)$ furnishes the existence of two waves: the first sound (or pressure wave) in which density and velocity vibrate with velocity $V_{1}:=\frac{\omega_{1,2}}{k_{r}}=\sqrt{p_{\rho}}(\omega$ real), and the second sound (or temperature wave) in which temperature and heat flux vibrate with velocity

$$
w^{2}=\left(\frac{\omega}{k_{r}}\right)^{2}=V_{2}^{2}-\frac{B^{\prime \prime 2} \kappa L_{R}^{2}}{4 V_{2}^{2} k_{r}^{2}+B^{\prime \prime 2} \kappa L_{R}^{2}} \quad \text { and } \quad k_{s}=\frac{w B^{\prime \prime} \kappa L_{R}}{2 V_{2}^{2}},
$$

where $V_{2}^{2}=\frac{\zeta}{\rho c_{V}}$ is the velocity of the second sound in the absence of vortices and $k_{s}$ is the attenuation. The longitudinal modes are

\begin{tabular}{|l|l|}
\hline$\omega_{1,2}= \pm k_{r} V_{1}$ & $\omega_{3,4}= \pm \sqrt{\frac{4 V_{2}^{4} k_{r}^{4}}{4 V_{2}^{2} k_{r}^{2}+B^{\prime \prime 2} \kappa L_{R}^{2}}}$ \\
\hline \hline$\tilde{\rho}=\psi$ & $\tilde{\rho}=0$ \\
$\tilde{v}_{1}= \pm \frac{V_{1}}{\rho} \psi$ & $\tilde{v}_{1}=0$ \\
$\tilde{T}_{0}=0$ & $\tilde{T}=T_{0} \psi$ \\
$\tilde{q}_{1}=0$ & $\tilde{q}_{1}= \pm \rho c_{V} T_{0} \sqrt{\frac{4 V_{2}^{4} k_{r}^{4}}{4 V_{2}^{2} k_{r}^{2}+B^{\prime \prime 2} \kappa L_{R}^{2}}} \psi$ \\
\hline
\end{tabular}

Therefore, as observed in (Snyder \& Putney, 1966), when the wave is propagated parallel to the rotation axis, the longitudinal modes are influenced by the rotation only through the axial component of the mutual friction ( $B^{\prime \prime}$ coefficient).

On the contrary, the transversal modes $\left(\tilde{v}_{2}, \tilde{v}_{3}, \tilde{q}_{2}\right.$ and $\left.\tilde{q}_{3}\right)$ are influenced by the rotation. In fact, the ones of velocity $\mathbf{v}$ admit nontrivial solutions if and only if $\omega_{5,6}= \pm 2|\Omega|$, while the ones related to $\mathbf{q}$ require the following dispersion relation:

$$
\omega_{7,8}= \pm\left(2 \Omega-\frac{1}{2} \kappa L_{R} B^{\prime}\right)-\frac{i}{2} \kappa L_{R}\left(B-B^{\prime \prime}\right) .
$$

These transversal modes are influenced from both dissipative and nondissipative contributions $B, B^{\prime}$ and $B^{\prime \prime}$ in the interaction between quasi-particles and vortex lines (Peruzza \& Sciacca, 2007).

Second case: $\mathbf{n}$ orthogonal to $\Omega$.

In the case in which the direction of propagation of the waves (for instance along $x_{2}$ ) is orthogonal to the rotation axis (along $x_{1}$ ), the longitudinal and transversal modes do not evolve independently. The first sound is coupled with one of the two transversal modes in which velocity vibrates, whereas fields $v_{1}, T$ and $q$ do not vibrate. 


\begin{tabular}{|l|l|}
\hline$\omega_{1}=0$ & $\omega_{2,3} \simeq \pm K V_{1}+O\left(\Omega^{2}\right)$ \\
\hline \hline$\tilde{\rho}=\psi$ & $\tilde{\rho}=\psi$ \\
$\tilde{v}_{2}=0$ & $\tilde{v}_{2}=\frac{ \pm V_{1}}{\rho} \psi$ \\
$\tilde{v}_{3}=i \frac{K V_{1}^{2}}{2 \Omega \rho} \psi$ & $\tilde{v}_{3}=-\frac{2 i \Omega}{\rho K} \psi$ \\
\hline
\end{tabular}

Second sound is coupled with a transversal mode in which $T, q_{2}$ and $q_{3}$ vibrate. Neglecting the second-order terms in $\Omega$, the dispersion relation becomes:

$$
\left(-\omega-\frac{i}{2} \kappa L_{R}\left(B-B^{\prime \prime}\right)\right)\left[-\omega\left(-\omega-\frac{i}{2} \kappa L_{R}\left(B-B^{\prime \prime}\right)\right)-K^{2} V_{2}^{2}\right]=0 .
$$

For $\omega \in \Re$ and $K=k_{r}+i k_{s}$ complex, one gets the solution $\omega_{4}=0$, which represents a stationary mode; and two solutions which furnish the following phase velocity and attenuation coefficient of the temperature wave (approximated with respect to $\left.\left(B-B^{\prime \prime}\right) \kappa L_{R} / \omega\right)$ :

$$
\begin{aligned}
& w \simeq \pm V_{2}\left(1-\frac{\left(B-B^{\prime \prime}\right)^{2} \kappa^{2} L_{R}^{2}}{32 \omega^{2}}\right)+O\left(\frac{\left(B-B^{\prime \prime}\right)^{4} \kappa^{4} L_{R}^{4}}{\omega^{4}}\right), \\
& k_{S} \simeq \frac{\left(B-B^{\prime \prime}\right) \kappa L_{R}}{4 V_{2}}+O\left(\frac{\left(B-B^{\prime \prime}\right)^{3} \kappa^{3} L_{R}^{3}}{\omega^{2}}\right) .
\end{aligned}
$$

The corresponding modes are $\tilde{\rho}=\tilde{q}_{1}=\tilde{v}_{1}=\tilde{v}_{2}=\tilde{v}_{3}=0$ and

\begin{tabular}{|l|l|}
\hline$\omega_{4}=0$ & $\omega_{5,6} \simeq \pm k_{r} V_{2}\left(1-\frac{\left(B-B^{\prime \prime}\right)^{2} \kappa^{2} L_{R}^{2}}{32 \omega^{2}}\right)+O\left(\frac{\left(B-B^{\prime \prime}\right)^{3} \kappa^{3} L_{R}^{3}}{\omega^{2}}\right)$ \\
\hline \hline$\tilde{T}=-\frac{i\left(2 \Omega-\frac{1}{2} \kappa L_{R} B^{\prime}\right)}{\zeta K} \psi$ & $\tilde{T}=T_{0} \psi$ \\
$\tilde{q}_{2}=0$ & $\tilde{q}_{2}=\frac{T_{0} \zeta}{V_{2}}\left(1-\frac{\left(B-B^{\prime \prime}\right)^{2} \kappa^{2} L_{R}^{2}}{32 \omega^{2}}\right) \psi$ \\
$\tilde{q}_{3}=\psi$ & $\tilde{q}_{3}=\frac{i\left(2 \Omega-\frac{1}{2} \kappa L_{R} B^{\prime}\right) T_{0} \zeta\left(1-\frac{\left(B-B^{\prime \prime}\right)^{2} \kappa^{2} L_{R}^{2}}{32 \omega^{2}}\right)}{V_{2}\left[ \pm k_{r} V_{2}\left(1-\frac{\left(B-B^{\prime \prime}\right)^{2} \kappa^{2} L_{R}^{2}}{32 \omega^{2}}\right)-\frac{i}{2}\left(B-B^{\prime \prime}\right) \kappa L_{R}\right]} \psi$ \\
\hline
\end{tabular}

We note that in the mode of frequency $\omega_{4}=0$, only the transversal component of the heat flux is involved.

For $\omega=\omega_{r}+i \omega_{s}$ complex and $K \in \Re$, the first solution of the dispersion relation (25) becomes $\omega_{4}=-\frac{i}{2}\left(B-B^{\prime \prime}\right) \kappa L_{R}$. This first mode corresponds to an extremely slow relaxation phenomenon involving the temperature and the transversal component of the heat flux

$$
\begin{array}{|l|}
\hline \omega_{4}=-\frac{i}{2}\left(B-B^{\prime \prime}\right) \kappa L_{R} \\
\hline \tilde{\rho}=\tilde{v}_{1}=\tilde{v}_{2}=\tilde{v}_{2}=\tilde{v}_{3}=0 \\
\tilde{T}=-\frac{i\left(2 \Omega-\frac{1}{2} \kappa L_{R} B^{\prime}\right)}{\zeta K} \psi \\
\tilde{q}_{2}=0 \\
\tilde{q}_{3}=\psi \\
\hline
\end{array}
$$

which, when $\Omega \rightarrow 0$, converges to a stationary mode. 


\subsection{Counterflow in a cylindrical tube}

Here we apply the model proposed in Section 2 to study the superfluid turbulence, in a cylindrical channel filled with helium II and submitted to a longitudinal stationary heat flux; for simplicity we suppose that the vortex distribution is described as an isotropic tangle. This allows us to suppose that the microscopic vorticity $\vec{\omega}$ (hence the unit vector $\mathbf{s}^{\prime}$ ) is isotropically distributed, so that

$$
<\mathbf{U}-\mathbf{s}^{\prime} \otimes \mathbf{s}^{\prime}>=\frac{2}{3} \mathbf{U}
$$

while $\langle\omega\rangle$ depends on the average vortex line length $L$ per unit volume, through the simple proportionality law $\langle\omega\rangle=\kappa L$ and $\lambda=B / 2, \lambda^{\prime}=0$. As a consequence, the pressure tensor (14) takes the simplified form

$$
\mathbf{P}_{\omega}=\lambda \kappa L \frac{2}{3} \mathbf{U} \quad \Rightarrow \quad \vec{\sigma}_{q}^{H}=-K_{1} L \mathbf{q},
$$

where $K_{1}=\frac{1}{3} \kappa B$.

\subsubsection{Wave propagation in presence of thermal counterflow}

Consider a cylindrical channel filled with helium II, submitted to a longitudinal heat flux $\mathbf{q}_{0}$, exceeding the critical value $\mathbf{q}_{c}$. We refer now to the experimental device (Donnelly \& Swanson, 1986), (Donnelly, 1991) in which second sound is excited transversally with respect to the channel. In this case, the heat flux $\mathbf{q}$ can be written as $\mathbf{q}=\mathbf{q}_{0}+\mathbf{q}^{\prime}$, with $\mathbf{q}^{\prime}$ the contribution to the heat flux, orthogonal to $\mathbf{q}_{0}$, due to the temperature wave. Suppose that the longitudinal heat flux $\mathbf{q}_{0}$ down the channel is much greater than the perturbation $\mathbf{q}^{\prime}$. Under these hypotheses, neglecting second order terms in $\mathbf{q}^{\prime}$, the production term is linear in the perturbation $\mathbf{q}^{\prime}$.

To study the second sound attenuation in the experiment described above, we use simplified field equations, where all the nonlinear contributions are neglected. Under the above hypotheses, omitting also the thermal dilation, the linearized set of field equations read as

$$
\left\{\begin{array}{l}
\frac{\partial \rho}{\partial t}+\rho \frac{\partial v_{j}}{\partial x_{j}}=0, \\
\rho \frac{\partial v_{i}}{\partial t}+\frac{\partial p}{\partial x_{i}}=0, \\
\frac{\partial T}{\partial t}+\frac{1}{\rho c_{V}} \frac{\partial q_{j}}{\partial x_{j}}=0 \\
\frac{\partial q_{i}}{\partial t}+\zeta \frac{\partial T}{\partial x_{i}}=-\frac{1}{3} \kappa B L q_{i} .
\end{array}\right.
$$

A stationary solution of the system (30) is (Jou et al., 2002):

$$
\rho=\rho_{0}, \quad \dot{\mathbf{v}}=\mathbf{0}, \quad T=T\left(x_{1}\right)=T_{0}-\frac{\kappa B L}{3 \zeta} q_{0} x_{1}, \quad \mathbf{q}=\mathbf{q}_{0},
$$

where $x_{1}$ is the direction of the heat flux $\mathbf{q}=\mathbf{q}_{0}$. In order to study the propagation of harmonic plane waves in the channel, we look for solutions of the system (30) of the form (22) with $\Gamma_{0}=\left(\rho_{0}, \mathbf{0}, T\left(x_{1}\right), \mathbf{q}_{0}\right)$. The longitudinal modes are obtained projecting the vectorial equations for the small amplitudes of velocity and heat flux on the direction orthogonal to the wave front. It is observed that the first sound is not influenced by the thermal counterflow, while 
the velocity and the attenuation of the second sound are influenced by the presence of the vortex tangle. The results are (Peruzza \& Sciacca, 2007):

with $p_{\rho}$ standing for $\partial p / \partial \rho$ and:

$$
w_{1,2}= \pm \sqrt{p_{\rho}}
$$

$$
w_{3,4}= \pm V_{2} \sqrt{\left(1+\frac{k_{s}^{2} V_{2}^{2}}{\omega^{2}}\right)^{-1}} \simeq \pm V_{2}\left(1-k_{s}^{2} \frac{V_{2}^{2}}{2 \omega^{2}}\right), \quad k_{s}=\frac{1}{6} \kappa B L w .
$$

The transversal modes are obtained projecting the vectorial equations for the small amplitudes of velocity and heat flux on the wave front. The solutions of this equation are: $\omega_{5}=0$ and $\omega_{6}=\frac{i}{3} \kappa B L$. The mode $\omega_{5}=0$ is a stationary mode.

\subsection{Combined situation of rotating counterflow}

The combined situation of rotation and heat flux, is a relatively new area of research (Jou \& Mongiovì, 2004), (Mongiovì \& Jou, 2005), (Tsubota et al., 2004). The first motivation of this interest is that from the experimental observations one deduces that the two effects are not merely additive; in particular, for $\mathbf{q}$ or $\Omega$ high, the measured values of $L$ are always less than $L_{H}+L_{R}$ (Swanson et al., 1983).

Under the simultaneous influence of heat flux $\mathbf{q}$ and rotation speed $\Omega$, rotation produces an ordered array of vortex lines parallel to rotation axis, whereas counterflow velocity causes a disordered tangle. In this way the total vortex line is given by the superposition of both contributions so that the vortex tangle is anisotropic. Therefore, assuming that the rotation is along the $x_{1}$ direction $\Omega=(\Omega, 0,0)$ and isotropy in the transversal $\left(x_{2}-x_{3}\right)$ plane, for the vorticity tensor $\mathbf{P}_{\omega}$, in combined situation of counterflow and rotation, the following explicit expression is taken

$$
\mathbf{P}_{\omega}=\frac{B}{2} \kappa L\left\{\frac{2}{3}(1-D) \mathbf{U}+D\left[\left(1-\frac{B^{\prime \prime}}{B}\right)(\mathbf{U}-\hat{\mathbf{\Omega}} \otimes \hat{\mathbf{\Omega}})+\frac{B^{\prime}}{B} \mathbf{W} \cdot \hat{\mathbf{\Omega}}+2 \frac{B^{\prime \prime}}{B} \hat{\mathbf{\Omega}} \otimes \hat{\mathbf{\Omega}}\right]\right\},
$$

where $D$ is a parameter between 0 and 1 related to the anisotropy of vortex lines, describing the relative weight of the array of vortex lines parallel to $\Omega$ and the disordered tangle of counterflow (when $D=0$ we recover an isotropic tangle - right hand side of Eq. (30d) -, whereas when $D=1$ the ordered array - Eq. (17)). Assuming $b=\frac{1}{3}(1-D)+\frac{D B^{\prime \prime}}{B}$ and $c=\frac{B^{\prime} D}{B}$, the vorticity tensor (33) can be written as:

$$
\mathbf{P}_{\omega}=\frac{B}{2} \kappa L\left\{\left(\begin{array}{ccc}
2 b & 0 & 0 \\
0 & 1-b & 0 \\
0 & 0 & 1-b
\end{array}\right)+\left(\begin{array}{ccc}
0 & 0 & 0 \\
0 & 0 & c \\
0 & -c & 0
\end{array}\right)\right\}
$$

Note that the isotropy in the $x_{2}-x_{3}$ plane may only be assumed when both $\Omega$ and $\mathbf{V}_{n s}$ are directed along the $x_{1}$ axis. A more general situations was studied in (Jou \& Mongiovì, 2006).

\subsubsection{Wave propagation with simultaneous rotation and counterflow}

Substituting the expression (34) into the linearized set of field equations (15), it becomes 


$$
\left\{\begin{array}{l}
\frac{\partial \rho}{\partial t}+\rho \frac{\partial v_{j}}{\partial x_{j}}=0, \\
\rho \frac{\partial v_{i}}{\partial t}+\frac{\partial p}{\partial x_{i}}+2 \rho \Omega v_{j} W_{1 j i}=0, \\
\frac{\partial T}{\partial t}+\frac{1}{\rho c_{V}} \frac{\partial q_{j}}{\partial x_{j}}=0, \\
\frac{\partial q_{i}}{\partial t}+\zeta \frac{\partial T}{\partial x_{i}}+2 \Omega q_{j} W_{1 j i}=-\frac{B}{2} \kappa L\left\{2 b q_{1} \delta_{1 i}+\left[(1-b) q_{2}+c q_{3}\right] \delta_{2 i}+\left[(1-b) q_{3}-c q_{2}\right] \delta_{3 i}\right\}
\end{array},\right.
$$

A stationary solution of this system is:

$$
\rho=\rho_{0}, \quad \dot{\mathbf{v}}=\mathbf{0}, \quad \mathbf{q}=\mathbf{q}_{0} \equiv\left(q_{0}, 0,0\right), \quad T=T\left(x_{i}\right)=T_{0}-2 \frac{B \kappa L}{2 \zeta} b q_{0} \delta_{1 i} x_{i} .
$$

In order to study the propagation of harmonic plane waves, we look for solutions of (35) of the form (22), with $\Gamma_{0}=\left(\rho_{0}, 0, T\left(x_{i}\right), \mathbf{q}_{0}\right)$.

Now, we investigate two different cases: $\mathbf{n}$ parallel to $\Omega$ and $\mathbf{n}$ orthogonal to $\boldsymbol{\Omega}$; the latter is the only case for which experimental data exist (Swanson et al., 1983).

First case: $\mathbf{n}$ parallel to $\Omega$.

Let $x_{1}$ be the direction of the rotation axis and of the unit vector $\mathbf{n}$ orthogonal to the wave front. In this case the longitudinal and transversal modes evolve independently. In particular, we can observe that the first sound is not influenced by the presence of the vortex tangle $k_{S}^{(1)}=0$ and $\tilde{T}=0, \mathbf{q}=0$

$$
\begin{array}{|l|}
\hline \omega_{1,2}= \pm k_{r} V_{1} \\
\hline \hline \tilde{\rho}=\psi \\
\tilde{v}_{1}=\frac{V_{1}}{\rho} \psi \\
\hline
\end{array}
$$

whereas the second sound suffers an extra attenuation due to the vortex tangle. This is confirmed by the approximate solutions of the dispersion relation

$$
\begin{aligned}
& w_{3,4} \simeq \pm V_{2}\left(1-\frac{B^{2} \kappa^{2} L^{2} b^{2}}{8 \omega^{2}}\right)+O\left(\frac{B^{4} \kappa^{4} L^{4} b^{4}}{16 \omega^{4}}\right), \\
& k_{s}^{(2)} \simeq \frac{B \kappa L b}{2 V_{2}}+O\left(\frac{B^{3} \kappa^{3} L^{3} b^{3}}{8 \omega^{2}}\right) .
\end{aligned}
$$

where $\omega$ is assumed real and $K=k_{r}+i k_{s}$ complex. When $\Omega=0$ and $b=1 / 3$ the results of the Section 3.3 are obtained again.

Now, we study the transversal modes, corresponding to $\omega_{5,6}= \pm 2|\Omega|$; in this case $\tilde{\rho}=\tilde{T}=$ $\tilde{q}_{1}=\tilde{q}_{2}=\tilde{q}_{3}=\tilde{v}_{1}=0$ and 


$$
\begin{array}{|l|}
\hline \omega_{5,6}= \pm 2|\Omega| \\
\hline \hline \tilde{v}_{3}=\psi \\
\tilde{v}_{2}= \pm i \psi \\
\hline
\end{array}
$$

They correspond to extremely slow phenomena, which, when $\Omega \rightarrow 0$, tend to stationary modes. Finally, the dispersion relation

$$
\omega_{7,8}= \pm\left(2 \Omega-\frac{B}{2} \kappa L c\right)-i \frac{B}{2} \kappa L(1-b)
$$

corresponds to the vibration of only these fields

$$
\begin{aligned}
& \omega_{7,8}= \pm\left(2 \Omega-\frac{B}{2} \kappa L c\right)-i \frac{B}{2} \kappa L(1-b) \\
& \hline \hline \tilde{q}_{3}=\psi \\
& \tilde{q}_{2}= \pm i \psi
\end{aligned}
$$

From (36), (37) and (38) one may obtain the following quantities $L, b$ and $c$ :

$$
L=\frac{-\omega_{s} w+V_{2}^{2} k_{s}}{\kappa w B / 2}, \quad b=\frac{V_{2}^{2} k_{s}}{-\omega_{s} w+V_{2}^{2} k_{s}}, \quad c=\frac{-\omega_{r} w+2 \Omega w}{-\omega_{s} w+V_{2}^{2} k_{s}},
$$

where we have put $\omega_{7}=\omega_{r}+i \omega_{s}$.

The results of this section imply that measurement in a single direction are enough to give information on all the variables describing the vortex tangle.

Second case: $\mathbf{n}$ orthogonal to $\Omega$.

Now we assume that the direction of propagation of the waves is orthogonal to the rotation axis (axis $x_{1}$ ), i.e. for example, $\mathbf{n}=(0,1,0)$. In this case the longitudinal and the transversal modes do not evolve independently. In particular, the first sound is coupled with one of the two transversal modes in which velocity vibrates, while the second sound is coupled with a transversal mode in which heat flux vibrates.

Fields $\tilde{\rho}, \tilde{v}_{2}, \tilde{v}_{3}$ have the same solutions and the same dispersion relation to the case of pure rotation

$$
-\omega\left[\omega^{2}-4 \Omega^{2}-K^{2} p_{\rho}\right]=0 .
$$

The dispersion relation of fields $\tilde{T}, \tilde{q}_{2}, \tilde{q}_{3}$ is instead:

$$
\left(-\omega-i \gamma \frac{B}{2} \kappa L(1-b)\right)\left[\omega\left(-\omega-i \frac{B}{2} \kappa L(1-b)\right)+K^{2} V_{2}^{2}\right]+\omega\left(2 i \Omega-i \frac{B}{2} \kappa L c\right)^{2}=0 .
$$

Assuming $\omega \in \Re$ and $K=k_{r}+i k_{s}$ and in the hypothesis of small dissipation $\left(k_{r}^{2} \gg k_{s}^{2}\right)$, one obtains:

$$
k_{s}=\frac{B}{2} \kappa L(1-b)\left(\frac{2 w^{2}-V_{2}^{2}}{2 w V_{2}^{2}}\right),
$$




$$
\begin{aligned}
& \left(\frac{\omega}{k_{r}}\right)^{2}=0, \quad \text { and } \\
& \left(\frac{\omega}{k_{r}}\right)^{2}=w^{2}=V_{2}^{2} \frac{\left(\omega^{2}-\tilde{B}\right)}{\left(\omega^{2}+\tilde{A}\right)}=V_{2}^{2} \frac{1}{1-\frac{\left(2 \Omega-\frac{B}{2} \kappa L c\right)^{2}}{\omega^{2}+(B / 2)^{2} \kappa^{2} L^{2}(1-b)^{2}}}
\end{aligned}
$$

where $\tilde{A}=-\left[\left(2 \Omega-\frac{B}{2} \kappa L c\right)^{2}-\frac{B^{2}}{4} \kappa^{2} L^{2}(1-b)^{2}\right]$ and $\tilde{B}=-\frac{B^{2}}{4} \kappa^{2} L^{2}(1-b)^{2}$.

We can remark that the coefficients $\tilde{A}$ and $\tilde{B}$ are negative and that $w^{2} \geq V_{2}^{2}$ because $\omega^{2}+\tilde{A} \leq$ $\omega^{2}-\tilde{B}$ and, in particular, $w^{2}=V_{2}^{2}$ for $\Omega=\frac{B \kappa L c}{4}$.

Now, studying the transversal modes, i.e. that ones corresponding to non zero $\tilde{v}_{1}$ and $\tilde{q}_{1}$, we obtain $\omega_{7}=0$, which corresponds to a stationary mode, and

$$
\omega_{8}=-i B \kappa L b .
$$

Summarizing, also in this case measurements in a single direction are enough to given information on all the variables describing the vortex tangle, namely $L, b$ and $c$, from equations (42), (43) and (44)

$$
\begin{aligned}
& L=\frac{4 k_{s} w V_{2}^{2}-\omega_{s}\left(2 w-V_{2}^{2}\right)}{\left(2 w^{2}-V_{2}^{2}\right) B \kappa}, \quad b=-\frac{\omega_{s}\left(2 w^{2}-V_{2}^{2}\right)}{4 k_{s} w V_{2}^{2}-\omega_{s}\left(2 w-V_{2}^{2}\right)}, \\
& c=\frac{4 \Omega\left(2 w^{2}-V_{2}^{2}\right)-\sqrt{\left(1-V_{2}^{2}\right)\left(4 k_{r}^{2}\left(2 w^{2}-V_{2}^{2}\right)^{2}+16 k_{s}^{2} V_{2}^{4}\right)}}{4 k_{s} w V_{2}^{2}-\omega_{s}\left(2 w^{2}-V_{2}^{2}\right)},
\end{aligned}
$$

where we have put $\omega_{8}=i \omega_{s}$ and $\omega_{s}=-\kappa L b B$.

In this subsection we have analyzed wave propagation in the combined situation of rotation and counterflow with the direction $n$ orthogonal to $\Omega$. In (Swanson et al., 1983) authors experimented the same situation, but they didn't represent the attenuation neither the speed of the second sound but only the vortex line density $L$ as function of $\Omega$ and $V_{n s}$. Therefore, it is unknown how they plotted these graphics, which hypothesis they made and what the anisotropy considered. Instead, the results of these two subsections allow to know the spatial distribution of the vortex tangle simply by performing experiments on waves propagating orthogonally to $\Omega$ (equations (39)) or parallelly to $\Omega$ (equations (45)).

From the physical point of view it is interesting to note that our detailed analysis in this subsection shows that, in contrast to which one could intuitively expect, measurements in a single direction are enough to give information on all the variables describing the vortex tangle, namely $L, b$ and $c$, for instance, from one of (36)-(37) and (38) or of (42)-(43) and (44). This is not an immediate intuitive result.

\subsection{Comparison with the two-fluid model}

To compare the one-fluid model of liquid helium II in a non-inertial frame with the two-fluid one, we recall that in (Mongiovì, 1991), (Mongiovì, 1993) it is shown that the linearized field equations (3) can be identified with those of the two-fluid non dissipative model if we define

$$
\zeta=\rho \frac{\rho_{s}}{\rho_{n}} T s^{2}
$$


and we make the following change of variables:

$$
\begin{gathered}
\mathbf{q}=\rho_{s} T_{s} \mathbf{V}_{n s}, \\
\mathbf{v}=\frac{\rho_{s}}{\rho} \mathbf{v}_{s}+\frac{\rho_{n}}{\rho} \mathbf{v}_{n},
\end{gathered}
$$

where, we recall, $\mathbf{v}_{n}$ and $\mathbf{v}_{s}$ are the mesoscopic velocities of the normal and superfluid components and $\mathbf{V}_{n s}=\mathbf{v}_{n}-\mathbf{v}_{s}$ is the counterflow velocity.

If we perform in the field equations (15) the change of variables (47-48), we check immediately that the first three equations are identical to the ones of the two-fluid model for helium II, even in non-inertial frame (Peruzza \& Sciacca, 2007). We concentrate therefore on the field equation for the heat flux. To the first order approximation with respect to the relative velocity $\mathbf{V}_{n s}$ and the derivatives of the field variables, we obtain:

$$
\frac{\partial \mathbf{V}_{n s}}{\partial t}+\frac{\zeta}{\rho_{s} T s} \nabla T+2 \Omega \times \mathbf{V}_{n s}=\frac{1}{\rho_{s} T s} \vec{\sigma}_{q},
$$

where $\vec{\sigma}_{q}$ stands for $\vec{\sigma}_{q}^{R}$ in rotation case, $\vec{\sigma}_{q}^{H}$ in counterflow case and $\vec{\sigma}_{q}^{H R}$ in rotating counterflow. We multiply equation (49) by $\rho_{n} / \rho$ and add it to the balance equation (15 b). Making use of the result $\mathbf{v}_{s}=\mathbf{v}-\left(\rho_{n} / \rho\right) \mathbf{V}_{n s}$, we find

$$
\frac{\partial \mathbf{v}_{s}}{\partial t}-s \nabla T+\frac{1}{\rho} \nabla p+2 \Omega \times \mathbf{v}_{s}+\frac{\rho_{n}}{\rho} \frac{1}{\rho_{s} T s} \vec{\sigma}_{q}=0 .
$$

In virtue of equation $d \mu=(1 / \rho) d p-s d T$, which relates the chemical potential $\mu=\epsilon-T s+$ $(p / \rho)$ to the equilibrium variables, the field equation for the superfluid velocity takes the form

$$
\rho_{s} \frac{\partial \mathbf{v}_{s}}{\partial t}+\rho_{s} \nabla \mu+2 \rho_{s} \Omega \times \mathbf{v}_{s}+\frac{\rho_{n}}{\rho} \frac{1}{T s} \vec{\sigma}_{q}=0 .
$$

Expression (51) is identical to the corresponding field equation for $\mathbf{v}_{s}$, obtained in the two-fluid model. Of course in the pure counterflow case $\Omega$ has to be set zero in (51). This result is a confirmation of the results derived in the framework of the one-fluid model based on E.T.. In counterflow experiments, equation (51) can be written as:

$$
\rho_{s} \frac{\partial \mathbf{v}_{s}}{\partial t}+\rho_{s} \nabla \mu=\mathbf{F}_{n s}^{(E)}, \quad \text { where } \quad \mathbf{F}_{n s}^{(E)}=\frac{1}{3} \frac{\rho_{s} \rho_{n}}{\rho} \kappa B \gamma^{2} V_{n s}^{2} \mathbf{V}_{n s}
$$

and relation $L=\gamma^{2} V_{n s}^{2}$ has been used.

To interpret the experimental results on stationary helium flow through channels using the two-fluid model, Gorter and Mellink (Gorter \& Mellink, 1949) and Vinen (Vinen, 1957) postulate the existence, in the field equation for the superfluid component, of a dissipative term proportional to the cube of the relative velocity $\mathbf{V}_{n s}$ :

$$
\mathbf{F}_{n s}^{(G M)}=\rho_{s} \rho_{n} \bar{A} V_{n s}^{2} \mathbf{V}_{n s},
$$

$\bar{A}$ being a temperature dependent coefficient. It is interesting to note that, setting $\bar{A}=$ $\kappa B \gamma^{2} /(3 \rho)$ in (52b), and using (47), the results of the present work are in full agreement with those of Gorter and Mellink. 


\section{Hydrodynamical model of inhomogeneous superfluid turbulence}

In Section 3 a first model of superfluid turbulence was presented, where the vortices were modeled through the pressure tensor $\mathbf{P}_{\omega}$ for which a constitutive relation was written.

Experiments (Vinen, 2000), (Vinen \& Niemela, 2002), show the formation of a new type of superfluid turbulence, which has some analogies with classical one, as for instance using towed or oscillating grids, or stirring liquid helium by means of propellers. In this situation (named co-flow) both components, normal and superfluid, flow along the same direction. To describe these experiments it is necessary to build up a hydrodynamic model of quantum turbulence, in which the interactions between both fields can be studied and the role of inhomogeneities is explicitly taken into account (Mongiovì \& Jou, 2007), (Ardizzone \& Gaeta, 2009).

In a more complete hydrodynamic model of superfluid turbulence the line density $L$ acquires field properties: it depends on the coordinates, it has a drift velocity $\mathbf{v}_{L}$, and it has associated a diffusion flux. These features are becoming increasingly relevant, as the local vortex density may be measured with higher precision, and the relative motion of vortices is observed and simulated. Thus it is important to describe situations going beyond the usual description of the vortex line density averaged over the volume. Our aim, in this Section, is to formulate a hydrodynamical framework sufficiently general to encompass vortex diffusion and to describe the interactions between the second sound waves and the vortices, instead of considering the latter as a rigid framework where such waves are simply dissipated. This is important because second sound provides the standard method of measuring the vortex line density $L$, and the mentioned dynamical mutual interplay between second sound and vortex lines may modify the standard results.

\subsection{The line density and Vinen's equation}

The most well known equation in the field of superfluid turbulence is Vinen's equation (Vinen, 1958), which describes the evolution of $L$, the total length of vortex lines per unit volume, in counterflow situations characterized by a heat flux $q$. Vinen suggested that in homogeneous counterflow turbulence there is a balance between generation and decay processes, which leads to a steady state of quantum turbulence in the form of a self-maintained vortex tangle. The Vinen's equation (2), written in terms of the variable $q$, is:

$$
\frac{d L}{d t}=\alpha_{q}|q| L^{3 / 2}-\beta_{q} L^{2}
$$

with $\alpha_{q}=\alpha_{v} \rho_{s} s T$ and $\beta_{q}=\kappa \beta_{v}$.

Vinen considered homogeneous superfluid turbulence and assumed that the time derivative $d L / d t$ is composed of two terms:

$$
\frac{d L}{d t}=\left[\frac{d L}{d t}\right]_{f}-\left[\frac{d L}{d t}\right]_{d}
$$

the first is responsible for the growth of $L$, the second for its decay. Vinen assumes that the production term $[d L / d t]_{f}$ depends linearly on the instantaneous value of $L$ and the force $\mathbf{f}$ between the vortex line and the normal component, which is linked to the modulus $|q|$ of the heat flux, and he obtained:

$$
\left[\frac{d L}{d t}\right]_{f}=\alpha_{v} \kappa V_{n s} L^{3 / 2}=\alpha_{q}|q| L^{3 / 2} .
$$


The form of the term responsible for the vortex decay was determined assuming Feynman's model of vortex breakup, analogous to Kolmogorov's cascade in classical turbulence

$$
\left[\frac{d L}{d t}\right]_{d}=-\beta_{v} \kappa L^{2}=-\beta_{q} L^{2}
$$

thus obtaining equation (54). A microscopic derivation of this equation was made by Schwarz (Schwarz, 1988).

The stationary solutions of this equation are $L=0$ and $L^{1 / 2}=\left(\alpha_{q} / \beta_{q}\right)|q|$. The non-zero solution is proportional to the square of the heat flux and describes well the full developed turbulence.

\subsection{Derivation of the hydrodynamical model}

The starting point here is to formulate a theory for a turbulent superfluid, which uses the averaged vortex line density $L$ in addition to the fields $\rho, \mathbf{v}, E$ and $\mathbf{q}$, used in Sections 2 and 3 . Because we want to formulate a general nonlinear theory, we will suppose that the dynamics of the excitations is described by a vector field $m_{i}$, which must be considered as an internal variable, linked to the heat flux $q_{i}$ through a constitutive relation, but not identical to it.

We consider for the fields $\rho, \mathbf{v}, E$ and $\mathbf{m}$ and $L$ the following balance equations written in terms of the non-convective terms (Ardizzone \& Gaeta, 2009):

$$
\left\{\begin{array}{l}
\dot{\rho}+\rho \frac{\partial v_{k}}{\partial x_{k}}=0, \\
\rho \dot{v}_{i}+\frac{\partial J_{i k}^{v}}{\partial x_{k}}=0 \\
\dot{E}+E \frac{\partial v_{k}}{\partial x_{k}}+\frac{\partial q_{k}}{\partial x_{k}}+J_{i k}^{v} \frac{\partial v_{i}}{\partial x_{k}}=0 \\
\dot{m}_{i}+m_{i} \frac{\partial v_{k}}{\partial x_{k}}+\frac{\partial J^{m} i k}{\partial x_{k}}=\sigma_{i}^{m}, \\
\dot{L}+L \frac{\partial v_{k}}{\partial x_{k}}+\frac{\partial J_{k}^{L}}{\partial x_{k}}=\sigma^{L} .
\end{array}\right.
$$

where $J_{i j}^{v}$ is the stress tensor, $J_{i j}^{m}$ the flux of the field $m_{i}$, and $J_{i}^{L}$ the flux of vortex lines; $\sigma_{i}^{m}$ and $\sigma^{L}$ are terms describing the net production of the field $m_{i}$ characterizing the dynamics of the excitations and the production of vortices. Dot denotes the material time derivative.

Since in the system (58) there are more unknowns than equations, it is necessary to complete it by adding constitutive equations, relating the variables $m_{i}, J_{i k^{\prime}}^{v}, J_{i k}^{m}$ and $J_{i}^{L}$ to the independent fields $\rho, E, q_{i}$ and $L$. As a consequence of the material objectivity principle, the constitutive equations can be expressed in the form:

$$
\begin{aligned}
& m_{i}=\alpha\left(\rho, E, q^{2}, L\right) q_{i}, \\
& J_{i k}^{v}=p\left(\rho, E, q^{2}, L\right) \delta_{i k}+a\left(\rho, E, q^{2}, L\right) q_{<i} q_{k>\prime}, \\
& J_{i k}^{m}=\beta\left(\rho, E, q^{2}, L\right) \delta_{i k}+\gamma\left(\rho, E, q^{2}, L\right) q_{<i} q_{k>}, \\
& J_{i}^{L}=v\left(\rho, E, q^{2}, L\right) q_{i} .
\end{aligned}
$$


where $\alpha, \beta, a, p, \gamma, v$ are scalar functions, $\delta_{i k}$ is the Kronecker symbol and $q_{<i} q_{k>}=q_{i} q_{k}-$ $\frac{1}{3} q^{2} \delta_{i k}$ is the deviatoric part of the diadic product $q_{i} q_{j}$.

\subsubsection{Restrictions imposed by the entropy principle}

Further restrictions on these constitutive relations are deduced from the second law of thermodynamics. Accordingly, there exists a convex function $S=S\left(\rho, E, q^{2}, L\right)$, the entropy per unit volume, and a vector function $J_{k}^{S}=\phi\left(\rho, E, q^{2}, L\right) q_{k}$, the entropy flux density, such that the rate of production of entropy $\sigma^{S}$ is non-negative

$$
\sigma^{S}=\dot{S}+S \frac{\partial v_{k}}{\partial x_{k}}+\frac{\partial J_{k}^{S}}{\partial x_{k}} \geq 0 .
$$

Note that this inequality does not hold for any value of the fundamental variables, but only for the thermodynamic processes, i.e. only for those values which are solution of the system (58). This means that we can consider the equations (58) as constraints for the entropy inequality to hold. A way to take these constraints into account was proposed by Liu (Liu, 1972): he showed that the entropy inequality becomes totally arbitrary provided that we complement it by the evolution equations for the fields $\rho, v_{i}, E, m_{i}$ and $L$ affected by Lagrange multipliers: $\Lambda_{\rho}=\Lambda_{\rho}\left(\rho, E, q^{2}, L\right), \Lambda_{i}^{v}=\Lambda_{v}\left(\rho, E, q^{2}, L\right) q_{i}, \Lambda_{E}=\Lambda_{E}\left(\rho, E, q^{2}, L\right), \Lambda_{i}^{m}=\lambda\left(\rho, E, q^{2}, L\right) q_{i}, \Lambda_{L}=$ $\Lambda_{L}\left(\rho, E, q^{2}, L\right)$. One obtains the following inequality, which is satisfied for arbitrary values of the field variables:

$$
\begin{aligned}
\dot{S}+S \frac{\partial v_{k}}{\partial x_{k}}+\frac{\partial J_{k}^{S}}{\partial x_{k}} & -\Lambda_{\rho}\left[\dot{\rho}+\rho \frac{\partial v_{k}}{\partial x_{k}}\right]-\Lambda_{i}^{v}\left[\dot{v}_{i}+\frac{1}{\rho} \frac{\partial J_{i k}^{v}}{\partial x_{k}}\right] \\
& -\Lambda_{E}\left[\dot{E}+E \frac{\partial v_{k}}{\partial x_{k}}+\frac{\partial q_{k}}{\partial x_{k}}+J_{i k}^{v} \frac{\partial v_{i}}{\partial x_{k}}\right] \\
& -\Lambda_{i}^{m}\left[\dot{m}_{i}+m_{i} \frac{\partial v_{k}}{\partial x_{k}}+\frac{\partial J_{i k}^{m}}{\partial x_{k}}-\sigma_{i}^{m}\right] \\
& -\Lambda_{L}\left[\dot{L}+L \frac{\partial v_{k}}{\partial x_{k}}+\frac{\partial J_{k}^{L}}{\partial x_{k}}-\sigma^{L}\right] \geq 0 .
\end{aligned}
$$

Imposing that the coefficients of the time derivatives of $\rho, v_{i}, E, q_{i}$ and $L$ vanish, one gets: $\Lambda_{v}=0$ and

$$
d S=\Lambda_{\rho} d \rho+\Lambda_{E} d E+\Lambda_{L} d L+\Lambda_{i}^{m} d m_{i}
$$

Imposing that the coefficients of space derivatives of $\rho, E, q_{i}$ and $L$ vanish, one finds:

$$
d J_{k}^{S}=\Lambda_{i}^{m} d J_{i k}^{m}+\Lambda_{L} d J_{k}^{L}+\Lambda_{E} d q_{k}
$$

From these relations in (Ardizzone et al., 2009) we have found:

$$
\begin{aligned}
& \Lambda_{v}=0, \quad a=0, \\
& d S=\Lambda_{\rho} d \rho+\Lambda_{E} d E+\lambda q_{i} d\left(\alpha q_{i}\right)+\Lambda_{L} d L \\
& S-\rho \Lambda_{\rho}-\Lambda_{E}(E+p)-\lambda \alpha q^{2}-\Lambda_{L} L=0
\end{aligned}
$$




$$
\begin{aligned}
& d \phi=\lambda\left(d \beta+\frac{1}{6} \gamma d q^{2}+\frac{2}{3} q^{2} d \gamma\right)+\Lambda_{L} d v \\
& \phi=\Lambda_{E}+\lambda \gamma q^{2}+\Lambda_{L} v .
\end{aligned}
$$

We note that all the relations (65)-(68) are exact, because no approximation has been used for their determination and maintain their validity also far from equilibrium.

It remains the following residual inequality for the entropy production:

$$
\sigma^{S}=\Lambda_{i}^{m} \sigma_{i}^{m}+\Lambda^{L} \sigma^{L} \geq 0
$$

Introducing the specific internal energy $\epsilon=E / \rho$, substituting the constitutive equations (59) in system (58) and the restriction $a=0$, the following system of field equations is obtained:

$$
\left\{\begin{array}{l}
\dot{\rho}+\rho \frac{\partial v_{k}}{\partial x_{k}}=0, \\
\rho \dot{v}_{i}+\frac{\partial p}{\partial x_{i}}=0, \\
\rho \dot{\epsilon}+\frac{\partial q_{k}}{\partial x_{k}}+p \frac{\partial v_{k}}{\partial x_{k}}=0, \\
\overbrace{\alpha q_{i}}+\alpha q_{i} \frac{\partial v_{j}}{\partial x_{j}}+\frac{\partial\left[\beta \delta_{i k}+\gamma q_{<i} q_{k>}\right]}{\partial x_{k}}=\sigma_{i}^{m}, \\
\dot{L}+L \frac{\partial v_{k}}{\partial x_{k}}+\frac{\partial\left(v q_{k}\right)}{\partial x_{k}}=\sigma^{L} .
\end{array}\right.
$$

Observe that in these equations there are the unknown quantities $\alpha, p, \epsilon, \beta, \gamma$ and $v$, which are not independent, because they must satisfy relations (65)-(68), and the productions $\sigma_{i}^{m}$ and $\sigma^{L}$ which must satisfy inequality (69).

In (Ardizzone et al., 2009) it is shown that, using a Legendre transformation, the constitutive theory is determined by the choice of only two scalar functions $S^{\prime}$ and $\phi^{\prime}$ of the intrinsic Lagrange multipliers, defined as:

$$
\begin{aligned}
& S^{\prime}=-S+\Lambda_{\rho} \rho+\Lambda_{E} E+\Lambda_{L} L+\Lambda_{i}^{m} m_{i}, \\
& \Phi_{k}^{\prime}=\phi^{\prime} \Lambda_{k}^{m}=-J_{k}^{S}+\Lambda_{E} q_{k}+\Lambda_{L} J_{k}^{L}+\Lambda_{i}^{m} J_{i k}^{m}
\end{aligned}
$$

Furthermore, if one chooses as state variables the fields

$$
\tilde{\Lambda}_{\rho}=\Lambda_{\rho}+\frac{1}{2} \Lambda_{E} v^{2}, \tilde{\Lambda}_{v_{i}}=-\Lambda_{E} v_{i}, \tilde{\Lambda}_{m_{i}}=\Lambda_{m_{i}}, \tilde{\Lambda}_{E}=\Lambda_{E}, \quad \tilde{\Lambda}_{L}=\Lambda_{L},
$$

the system of field equation (58) assumes the form of a symmetric hyperbolic system and, therefore, for it the Cauchy problem is well posed, i.e. the existence, uniqueness and continuous dependence of its solutions by the initial data is assured.

\subsubsection{Physical interpretation of the constitutive quantities and of the Lagrange multipliers} As shown, the use of the Lagrange multipliers as independent variables results very useful from a mathematical point of view. In order to single out the physical meaning of the constitutive quantities and of the Lagrange multipliers, we analyze now in detail the relations 
obtained in the previous section. First we will determine the equilibrium values for these multipliers. Denoting with $\mathrm{Y}$ any of the scalar quantities $\alpha, h, \phi, p, \beta, \gamma, v, \Lambda_{\rho}, \Lambda_{E}, \lambda, \Lambda_{L}$ and making the position

$$
\mathrm{Y}_{0}\left(\rho, E, q^{2}, L\right)=\mathrm{Y}_{0}(\rho, E, L)+O\left(q^{2}\right),
$$

the following relations are obtained:

$$
\begin{aligned}
& d S_{0}=\Lambda_{0}^{\rho} d \rho+\Lambda_{0}^{E} d E+\Lambda_{0}^{L} d L, \\
& S_{0}-\rho \Lambda_{0}^{\rho}-\Lambda_{0}^{E}\left(E+p_{0}\right)-\Lambda_{0}^{L} L=0 \\
& d \phi_{0}=\lambda_{0} d \beta_{0}+\Lambda_{0}^{L} d v_{0}, \\
& \phi_{0}=\Lambda_{0}^{E}+\Lambda_{0}^{L} v_{0} .
\end{aligned}
$$

Introduce now a "generalized temperature" as the reciprocal of the first-order part of the Lagrange multiplier of the energy

$$
\Lambda_{0}^{E}=\left[\frac{\partial S_{0}}{\partial E}\right]_{\rho, L}=\frac{1}{T}
$$

and observe that, in the laminar regime (when $L=0$ ), $\Lambda_{0}^{E}$ reduces to the absolute temperature of thermostatics. In the presence of a vortex tangle the quantity (76) depends also on the line density $L$. Writing equations (75a) and (75b) as

$$
\begin{gathered}
d E=T d S_{0}-T \Lambda_{0}^{\rho} d \rho-T \Lambda_{0}^{L} d L, \\
-T \Lambda_{0}^{\rho}=\frac{E}{\rho}-T \frac{S_{0}}{\rho}+\frac{p_{0}+L T \Lambda_{0}^{L}}{\rho},
\end{gathered}
$$

and defining the quantity $-\Lambda_{0}^{\rho} / \Lambda_{0}^{E}=-T \Lambda_{0}^{\rho}$ as the "mass chemical potential" in turbulent superfluid

$$
-T \Lambda_{0}^{\rho}=-T\left[\frac{\partial S_{0}}{\partial \rho}\right]_{E, L}=\mu_{0}^{\rho},
$$

and the quantity $-\Lambda_{0}^{L} / \Lambda_{0}^{E}=-T \Lambda_{0}^{L}$ as the "chemical potential of vortex lines", which is denoted with $\mu_{0}^{L}$,

$$
-T \Lambda_{0}^{L}=-T\left[\frac{\partial S_{0}}{\partial L}\right]_{\rho, L}=\mu_{0}^{L},
$$

one can write equations (77) and (78) in the following form:

$$
\begin{gathered}
d S_{0}=\frac{1}{T} d E-\frac{1}{T} \mu_{0}^{\rho} d \rho-\frac{1}{T} \mu_{0}^{L} d L, \\
\rho \mu_{0}^{\rho}+L \mu_{0}^{L}=E-T h_{0}+p_{0} .
\end{gathered}
$$

Indeed, in absence of vortices ( $L=0$ ) equation (77) is just Gibbs equation of thermostatics and the quantity (79) is the equilibrium chemical potential. The presence of vortices modifies the energy density $E$, and introduce a new chemical potential. 
Consider now the consequences of equations (75c) and (75d) which concern the expressions of the fluxes. Using definitions (76) and (80), we get:

$$
\lambda_{0} d \beta_{0}=d\left(\frac{1}{T}\right)-v_{0} d\left(\frac{\mu_{0}^{L}}{T}\right) .
$$

From this equation, recalling that in (Mongiovì \& Jou, 2007) it has shown that $\mu_{0}^{L}$ depends only on $T$ and $L$, one obtains $\partial \beta_{0} / \partial \rho=0$ and

$$
\frac{\partial \beta_{0}}{\partial T}=\zeta_{0}=-\frac{1}{T^{2} \lambda_{0}}\left[1+v_{0} T^{2} \frac{\partial}{\partial T}\left(\frac{\mu_{0}^{L}}{T}\right)\right], \quad \frac{\partial \beta_{0}}{\partial L}=\chi_{0}=-\frac{v_{0}}{T \lambda_{0}} \frac{\partial \mu_{0}^{L}}{\partial L} .
$$

In (Mongiovì \& Jou, 2007) it was shown also that it results $\lambda_{0}<0, \zeta_{0} \geq 0, v_{0} \leq 0$ and $\chi_{0} \leq 0$.

\subsubsection{The constitutive relations far from equilibrium}

Finally, we analyze the complete mathematical expressions far from equilibrium of the constitutive functions and of the Lagrange multipliers.

Non-equilibrium temperature. First, we introduce the following quantity:

$$
\theta=\frac{1}{\Lambda_{E}\left(\rho, E, L, q^{2}\right)}
$$

which, near equilibrium $\left(L=0, q_{i}=0\right)$ can be identified with the local equilibrium absolute temperature. In agreement with (Jou et al., 2001), we will call $\theta$ "non-equilibrium temperature", a topic which is receiving much attention in current non-equilibrium Thermodynamics (Casas-Vázquez \& Jou, 2003).

Using this quantity, the scalar potential $S^{\prime}$ is expressed as:

$$
S^{\prime}=-\frac{p}{\theta}
$$

Non-equilibrium Chemical Potentials. As we have seen, at equilibrium the quantities $-\Lambda_{\rho} / \Lambda_{E}$ and $-\Lambda_{L} / \Lambda_{E}$ can be interpreted as the equilibrium mass chemical potential and the equilibrium vortex line density chemical potential. Therefore, we define as non-equilibrium chemical potentials the quantities:

$$
\mu_{\rho}=-\frac{\Lambda_{\rho}}{\Lambda_{E}}, \quad \text { and } \quad \mu_{L}=-\frac{\Lambda_{L}}{\Lambda_{E}} .
$$

Generalized Gibbs equation. Using equations (65) and (66) and defining $s=S / \rho$ the non-equilibrium specific entropy, one obtains

$$
\begin{gathered}
\theta d(\rho s)=d E-\mu_{\rho} d \rho-\mu_{L} d L+\theta \lambda q_{i} d\left(\alpha q_{i}\right), \\
\mu_{\rho}+\frac{L}{\rho} \mu_{L}=\epsilon-\theta s+\frac{p}{\rho}+\frac{\theta}{\rho} \alpha \lambda q^{2} .
\end{gathered}
$$

One gets also:

$$
d p=\rho d \mu_{\rho}+L d \mu_{L}+\rho s d \theta-\alpha q_{i} d\left(\theta \lambda q_{i}\right) .
$$

For the interested reader, in (Ardizzone \& Gaeta, 2009), the complete constitutive theory can be found. 
Non-equilibrium Entropy Flux. The theory developed here furnishes also the complete non-equilibrium expression of the entropy flux $J_{k}^{S}$. Remembering relation (68), we can write:

$$
J_{i}^{S}=\left(\frac{1}{\theta}+v \Lambda_{L}+\gamma \lambda q^{2}\right) q_{i}=\frac{1}{\theta}\left(q_{i}-\mu_{L} J_{i}^{L}+\theta \gamma \lambda q^{2} q_{i}\right) .
$$

This equation shows that, in a nonlinear theory of Superfluid Turbulence, the entropy flux is different from the product of the reciprocal non-equilibrium temperature and the heat flux, but it contains additional terms depending on the flux of heat flux and on the flux of line density.

\subsection{Linearized field equations}

Now we will apply the general set of equations derived to the analysis of two specific situations: vortex diffusion and wave propagation. First of all, we note that, substituting in (70) the constitutive expressions obtained in Subsection 4.2.2, and neglecting nonlinear terms in the fluxes, the following system is obtained (Mongiovì \& Jou, 2007):

$$
\left\{\begin{array}{l}
\dot{\rho}+\rho \nabla \cdot \mathbf{v}=0, \\
\rho \dot{\mathbf{v}}+\nabla p_{0}=0, \\
\rho \dot{\epsilon}+\nabla \cdot \mathbf{q}+p_{0} \nabla \cdot \mathbf{v}=0, \\
\dot{\mathbf{q}}+\zeta_{0} \nabla \mathrm{T}+\chi_{0} \nabla L=\sigma^{\mathbf{q}}, \\
\dot{L}+L \nabla \cdot \mathbf{v}+\nabla \cdot\left(v_{0} \mathbf{q}\right)=\sigma^{L},
\end{array}\right.
$$

with $\zeta_{0}$ and $\chi_{0}$ defined by (84) and satisfying $\left(v_{0} / \chi_{0}\right)=-\left(T \lambda_{0}^{q} L / \epsilon_{V}\right)$.

The total pressure of the turbulent superfluid has the form (Mongiovì \& Jou, 2007):

$$
p_{0}=p^{*}+\epsilon_{V} L
$$

$p^{*}$ being the pressure of the bulk superfluid and $\epsilon_{V} L$ the contribution of the tangle, with $\epsilon_{V}$ the energy per unit length of the vortices (Donnelly, 1991).

For the production terms $\sigma^{\mathbf{q}}$ and $\sigma^{L}$, we will take

$$
\sigma^{\mathbf{q}}=-K_{1} L \mathbf{q} \quad \sigma^{L}=-\beta_{q} L^{2}+\alpha_{q}|q| L^{3 / 2},
$$

where $K_{1}=\frac{1}{3} \kappa B$. In this approximation, the unknown coefficients, which must be determined from experimental data, are the specific energy $\epsilon$, the pressure $p_{0}$, and the three coefficients $\zeta_{0}, \chi_{0}$ and $v_{0}$, which are functions only of $T$ and $L$. Here, we will focus a special attention on the coefficients $\chi_{0}$ and $v_{0}$, which are the new ones appearing in the present formulation, as compared with the formulation presented in (58).

\subsubsection{The drift velocity of the tangle}

As observed, in a hydrodynamical model of turbulent superfluids, the line density $L$ acquires field properties and its rate of change must obey a balance equation of the general form:

$$
\frac{\partial L}{\partial t}+\nabla \cdot\left(L \mathbf{v}^{L}\right)=\sigma^{L}
$$


with $\mathbf{v}^{L}$ the drift velocity of the tangle. If we now observe that the last equation of system (92) can be written:

$$
\frac{\partial L}{\partial t}+\nabla \cdot\left(L \mathbf{v}+v_{0} \mathbf{q}\right)=\sigma^{L},
$$

we conclude that the drift velocity of the tangle, with respect to the container, is given by

$$
\mathbf{v}^{L}=\mathbf{v}+\frac{v_{0}}{L} \mathbf{q} .
$$

Note that the velocity $\mathbf{v}^{L}$ does not coincide with the microscopic velocity of the vortex line element, but represents an averaged macroscopic velocity of this quantity. It is to make attention to the fact that often in the literature the microscopic velocity $\dot{\mathbf{s}}$ is denoted with $\mathbf{v}_{L}$.

Observing that in counterflow experiments $(\mathbf{v}=0)$ results $\mathbf{v}^{L}=v_{0} \mathbf{q} / L$, and recalling that measurements (in developed superfluid turbulence) show that the vortex tangle drifts as a whole toward the heater, we conclude that $v_{0} \leq 0$. The measurement of the drift velocity $\mathbf{v}^{L}$ of the vortex tangle, together with the measurement of $\mathbf{q}$ and $L$, would allow one to obtain quantitative values for the coefficient $v_{0}$. In the following section we will propose a way to measure the coefficient $\chi_{0}$ too.

Another possibility is to interpret $v_{0} \mathbf{q}=\mathbf{J}^{L}$ as the diffusion flux of vortices, which since $v_{0} \leq 0$, would be opposite to the direction of $\mathbf{q}$. Note that, in this model, if $\mathbf{q}=0, \mathbf{J}^{L}$ is also zero.

\subsubsection{Vortex diffusion}

An interesting physical consequence from the generalized equations (92) is the description of vortex diffusion. A diffusion equation for the vortex line density was proposed for the first time by van Beelen et al. (van Beelen et al., 1988), in an analysis of vorticity in capillary flow of superfluid helium, in situations with a step change in $L$ arising when the tube is divided in a region with laminar flow and another one with turbulent flow. Assume, for the sake of simplicity, that $T=$ constant and that $\mathbf{q}$ varies very slowly, in such a way that $\dot{\mathbf{q}}$ may be neglected. We find from (92d) and (94a) that

$$
\mathbf{q}=-\frac{\chi_{0}}{K_{1} L} \nabla L
$$

Introducing this expression in equation (92e), we find:

$$
\frac{d L}{d t}+L \nabla \cdot \mathbf{v}-\frac{v_{0} \chi_{0}}{K_{1}} \nabla \cdot\left(\frac{\nabla L}{L}\right)=\sigma^{L}=-\beta_{q} L^{2}+\alpha_{q} q L^{3 / 2},
$$

where $q$ denotes the modulus of (98). Equation (99) can be written (if $\nabla L \neq 0$ )

$$
\frac{d L}{d t}+L \nabla \cdot \mathbf{v}-\frac{v_{0} \chi_{0}}{K_{1} L} \Delta L+\left(\frac{v_{0} \chi_{0}}{K_{1} L^{2}}\right)(\nabla L)^{2}=\sigma^{L} .
$$

Then, we have for $L$ a reaction-diffusion equation, which generalizes the usual Vinen's equation (54) to inhomogeneous situations. The diffusivity coefficient is (Mongiovì \& Jou, 2007)

$$
D_{\text {diff }}=\frac{v_{0} \chi_{0}}{K_{1} L} .
$$

Since $K_{1}>0$, it turns out that $D_{\text {diff }}>0$, as it is expected. Thus, the vortices will diffuse from regions of higher $L$ to those of lower $L$. Note that $D_{\text {diff }}$ must have dimensions $(\text { length })^{2} /$ time, 
the same dimensions as $\kappa$. Then, a dimensional ansatz could be $D_{\text {diff }} \propto \kappa$. Indeed, Tsubota et al. (Tsubota et al., 2003b) have studied numerically the spatial vortex diffusion in a localized initial tangle allowed to diffuse freely, and they found for $D_{\text {diff }}$ at very low temperatures (when there is practically no normal fluid), a value $D_{\text {diff }} \approx(0.1 \pm 0.05) \kappa$.

If $\mathbf{v}$ vanishes, or if its divergence vanishes, equation (99), neglecting also the term in $(\nabla L)^{2}$, yields

$$
\dot{L}=-\beta_{q} L^{2}+\alpha_{q} q L^{3 / 2}+D_{\text {diff }} \Delta L
$$

Equation (102) indicates two temporal scales for the evolution of $L$ : one of them is due to the production-destruction term $\left(\tau_{\text {decay }}\right)$ and another one to the diffusion

$$
\tau_{\text {decay }} \approx\left[\beta_{q} L-\alpha_{q} q L^{1 / 2}\right]^{-1}, \quad \tau_{\text {diff }} \approx \frac{X^{2}}{D_{\text {diff }}},
$$

where $X$ is the size of the system. For large values of $L, \tau_{\text {decay }}$ will be much shorter and the production-destruction dynamics will dominate over diffusion; for small $L$, instead, diffusion processes may be dominant. This may be also understood from a microscopic perspective because the mean free path of vortex motion is of the order of intervortex spacing, of the order of $L^{-1 / 2}$, and therefore it increases for low values of $L$.

A more general situation for the vortex diffusion flux is to keep the temperature gradient in (92d). In this more general case, $\mathbf{q}$ is not more parallel to $\nabla L$ but results

$$
\mathbf{q}=-\frac{\chi_{0}}{K_{1} L} \nabla L-\frac{\zeta_{0}}{K_{1} L} \nabla T
$$

in which case, it is:

$$
\mathbf{J}^{L}=v_{0} \mathbf{q}=-D_{\text {diff }} \nabla L-D_{\text {diff }} \frac{\zeta_{0}}{\chi_{0}} \nabla T
$$

Thus, if $\nabla L=0$, (105) will yield

$$
\mathbf{q}=-\lambda_{e f f} \nabla T,
$$

with an effective thermal conductivity $\lambda_{e f f}=\frac{D_{\text {diff }} \zeta_{0}}{\chi_{0} v_{0}}>0$. As in the case of the diffusion coefficient $D_{\text {diff }}, \lambda_{\text {eff }}$ is expected to be positive. Note that $\lambda_{e f f}>0$ implies again $\chi_{0} v_{0}>0$. The second term in (105) plays a role analogous to thermal diffusion - or Soret effect — in usual diffusion of particles. In this case, equation (99) modifies as

$$
\frac{d L}{d t}+L \nabla \cdot \mathbf{v}-\frac{v_{0} \chi_{0}}{K_{1} L} \Delta L-\frac{v_{0} \zeta_{0}}{K_{1} L} \Delta T=\sigma^{L} .
$$

This kind of situations have not been studied enough in the context of vortex tangles, but they would arise in a natural way when trying to understand the behavior of quantum turbulence in the presence of a temperature gradient.

Expression (105) yields a coupling between the heat flux and an inhomogeneity in $L$; in other terms, it means that a heat flux may influence the vortex line density. In view of (104), we have

$$
-\chi_{0} \nabla L=K_{1} L \mathbf{q}+\zeta_{0} \nabla T
$$


From here, it follows that there should be a slight inhomogeneity in $L$ in such a way that $\nabla L$ points in the same direction as $K_{1} L \mathbf{q}+\zeta_{0} \nabla T$. It follows, in contrast with the standard assumption that the vortex line density is longitudinally homogeneous in counterflow experiments, that the vortex tangle would be slightly inhomogeneous. Thus, an experiment suggested by our formalism would be to carefully measure the longitudinal profile of $L$ along the heat flux, to check whether there is a slight increase in L. Furthermore, equation (108) would allow one to measure the coefficient $\chi_{0}$, in the linear approximation.

Since below (97) we have mentioned a way to measure $v_{0}$, it turns out that the new coefficients $v_{0}$ and $\chi_{0}$ could be measured independently of each other.

\subsubsection{Propagation of plane harmonic waves}

Here, we will study wave propagation in counterflow vortex tangles, assuming that only fields $T, \mathbf{q}$ and $L$ are involved. The equations for these fields, expressing the energy in terms of $T$ and $L$, are simply:

$$
\left\{\begin{array}{l}
\rho c_{V} \dot{T}+\rho \epsilon_{L} \dot{L}+\nabla \cdot \mathbf{q}=0, \\
\dot{\mathbf{q}}+\zeta_{0} \nabla T+\chi_{0} \nabla L=-K_{1} L \mathbf{q}, \\
\dot{L}+\nabla \cdot\left(v_{0} \mathbf{q}\right)=-\beta_{q} L^{2}+\alpha_{q} q L^{3 / 2} .
\end{array}\right.
$$

where $c_{V}=\partial \epsilon / \partial T$ is the specific heat at constant volume, $\epsilon_{L}=\partial \epsilon / \partial L \simeq \epsilon_{V}$.

These equations are enough for the discussion of the physical effects of the coupling of second-sound and the distortion of the vortex tangle (represented by the inhomogeneities in $L$ ), which must be taken into account in an analysis of the vortex tangle by means of second sound. In fact, some of the previous hydrodynamical analyses of turbulent superfluids had this problem as one of their main motivations (Tsubota et al., 2003a), (Tsubota et al., 2003b). As we can easily see, a stationary solution of system (109) is:

$$
\mathbf{q}=\mathbf{q}_{0}=\left(q_{10}, 0,0\right), \quad L=L_{0}=\frac{\alpha_{q}^{2}}{\beta_{q}^{2}}\left[q_{10}\right]^{2}, \quad T=T_{0}(\mathbf{x})=T^{*}-\frac{K_{1} L_{0} q_{10}}{\zeta_{0}} x_{1},
$$

with $q_{10}>0$.

To study the wave propagation in a neighborhood of this solution, we substitute $\sigma^{\mathbf{q}}$ and $\sigma^{L}$ with

$$
\begin{gathered}
\sigma^{\mathbf{q}} \simeq-K_{1}\left[L_{0} \mathbf{q}+\mathbf{q}_{0}\left(L-L_{0}\right)\right], \\
\sigma^{L} \simeq-\left[2 \beta_{q} L_{0}-\frac{3}{2} \alpha_{q} q_{10} L^{1 / 2}\right]\left(L-L_{0}\right)+\alpha_{q} L_{0}^{3 / 2} \hat{\mathbf{q}}_{0} \cdot\left(\mathbf{q}-\mathbf{q}_{0}\right),
\end{gathered}
$$

obtaining:

$$
\left\{\begin{array}{l}
\rho c_{V} \frac{\partial T}{\partial t}+\rho \epsilon_{L} \frac{\partial L}{\partial t}+\nabla \cdot \mathbf{q}=0 \\
\frac{\partial \mathbf{q}}{\partial t}+\zeta_{0} \nabla T+\chi_{0} \nabla L=-K_{1}\left[L_{0} \mathbf{q}+\mathbf{q}_{0}\left(L-L_{0}\right)\right] \\
\frac{\partial L}{\partial t}+v_{0} \nabla \cdot \mathbf{q}=-\left[2 \beta_{q} L_{0}-\frac{3}{2} \alpha_{q} q_{10} L^{1 / 2}\right]\left(L-L_{0}\right)+\alpha_{q} L_{0}^{3 / 2} \hat{\mathbf{q}}_{0} \cdot\left(\mathbf{q}-\mathbf{q}_{0}\right) .
\end{array}\right.
$$


Consider the propagation of harmonic plane waves, seeking for solutions of equations (109) of the form

$$
T=T_{0}(\mathbf{x})+\tilde{T} e^{i(k \mathbf{n} \cdot \mathbf{x}-\omega t)}, \quad \mathbf{q}=\mathbf{q}_{0}+\tilde{\mathbf{q}} e^{i(k \mathbf{n} \cdot \mathbf{x}-\omega t)}, \quad L=L_{0}+\tilde{L} e^{i(k \mathbf{n} \cdot \mathbf{x}-\omega t)},
$$

where $K=k_{r}+i k_{s}$ is the complex wave number, $\omega$ the real frequency and $\mathbf{n}$ the unit vector in the direction of the wave propagation. Furthermore, we suppose that the oversigned quantities denote small amplitudes, whose products can be neglected. Inserting (114) in the linearized field equations (109), and making the positions:

$$
N_{1}=K_{1} L_{0}, \quad N_{2}=2 \beta_{q} L_{0}-\frac{3}{2} \alpha_{q} L_{0}^{1 / 2} q_{10}, \quad N_{3}=K q_{10}, \quad N_{4}=\alpha_{q} q_{10} L_{0}^{3 / 2},
$$

we obtained in (Mongiovì \& Jou, 2007), when the wave is collinear with the heat flux $\mathbf{q}$, the following dispersion relation:

$$
\begin{aligned}
\omega^{2}=K^{2}[ & \left.V_{2}^{2}\left(1-\rho \epsilon_{L} v_{0}\right)+v_{0} \chi_{0}\right]+ \\
& \quad i \omega\left(N_{1}+N_{2}\right)+i \frac{K^{2}}{\omega} V_{2}^{2} N_{2}-i K\left[\left(\chi_{0}+V_{2}^{2} \rho \epsilon_{L}\right) N_{4}-v_{0} N_{3}\right],
\end{aligned}
$$

while, when the wave is orthogonal with the heat flux $\mathbf{q}$, we obtained

$$
\omega^{2}=K^{2}\left[V_{2}^{2}\left(1-\rho \epsilon_{L} v_{0}\right)+v_{0} \chi_{0}\right]+N_{1} N_{2}-i \omega\left(N_{1}+N_{2}\right)+i \frac{K^{2}}{\omega} V_{2}^{2}\left(N_{2}-\frac{N_{3} N_{4}}{\omega+i N_{1}}\right) .
$$

We compare these results with the result obtained in Section 3(see also (Jou et al., 2002)), where we supposed $L$ a fixed quantity, and the term $v_{0}$ was assumed to vanish, eliminating in this way the effects of the oscillations of $\mathbf{q}$ on the vortex line density $L$ of the tangle. Assuming $v_{0}=N_{2}=N_{4}=0$ in (116) and (117), the same dispersion relation for the second sound is obtained

$$
\omega^{2}=V_{2}^{2} K^{2}-i \omega K_{1} L_{0}
$$

Comparison of (116) and (117) with (118) shows that the distortion of the vortex tangle under the action of the heat wave, and its corresponding back reaction on the latter, implies remarkable changes in the velocity and the attenuation of the second sound, the latter effect depending on the relative direction between $\mathbf{q}_{0}$ and $\mathbf{n}$.

\subsubsection{High-frequency waves}

In the hypothesis of high-frequency waves, which means $\omega \gg N_{1}, \omega \gg N_{2}$ and $|\bar{K}| \gg$ $\max \left(\frac{K_{1} q_{10}}{\chi_{0}}, \frac{\alpha_{q} q_{10} L_{0}^{3 / 2}}{\nu_{0}}\right)$, the dispersion relation (n parallel or orthogonal to the initial heat flux) is

$$
w^{2}=V_{2}^{2}\left(1-v_{0} \rho \epsilon_{L}\right)+v_{\infty}^{2}
$$

where $w=\omega / k_{r}$ is the speed of the wave, $V_{2}^{2}=\zeta_{0} / \rho c_{V}$ is the second sound speed in the absence of vortices and

$$
v_{\infty}^{2}=\chi_{0} v_{0}
$$


is the speed of the vortex density wave, which was found in (Jou et al., 2007).

If we try to read the relation (119) in terms of the second sound, we note that the vortex vibrations modify this second sound speed through the two contributions $-V_{2}^{2} v_{0} \rho \epsilon_{L}$ and $v_{\infty}^{2}$, the latter due to the presence of the vortex density waves and the former due to the reciprocal existence of two waves. The correction for the speed of the second sound is not important, because $V_{2}$ is of the order of $20 \mathrm{~m} / \mathrm{s}$ near $1,7 \mathrm{~K}$, whereas, for $L_{0}=10^{6} \mathrm{~cm}^{-2}$, the speed of vortex density waves would be of the order $0,25 \mathrm{~cm} / \mathrm{s}$, much lower than $V_{2}$ (Jou et al., 2007). To obtain approximate solutions of equations (116) and (117), we assume that the quantities $N_{1}, N_{2}, N_{3}$ and $N_{4}$ are coefficients small enough to assume them as perturbations of the physical system. This is reasonable at high-frequencies, since we have assumed that $\omega \gg N_{1}$, $\omega \gg N_{2}$ and $|\bar{K}| \gg \max \left(\frac{K q_{10}}{\chi_{0}}, \frac{A q_{10} L_{0}^{3 / 2}}{v_{0}}\right)$.

Therefore, let assume that the speed of the wave has the following expression

$$
\tilde{w}=\frac{\omega}{k_{r}}=w+\delta
$$

for which substituting it in the equations (116) and (117), we obtain at the lowest order the relation (119) for the speed $w$. From the next order follows that $\delta=0$, that is the perturbations due to the coefficients $N_{1}, N_{2}, N_{3}$ and $N_{4}$ do not modify the speed of the wave while they modify the coefficients $k_{s}$ related to the attenuation in the form, which in the parallel case is

$$
k_{s}^{\|}=\frac{N_{2}\left(w^{2}-V_{2}^{2}\right)+w\left(\rho \epsilon_{L} N_{4} V_{2}^{2}+N_{1} w+N_{3} v_{0}-N_{4} \chi_{0}\right)}{2 w^{3}} .
$$

and in the orthogonal case is

$$
k_{s}^{\perp}=\frac{N_{2}\left(w^{2}-V_{2}^{2}\right)+N_{1} w^{2}}{2 w^{3}}
$$

From a comparison between the two relations of $k_{s}$, (122) and (123), one may note that

$$
k_{s}^{\|}=k_{s}^{\perp}+\frac{\left(\rho \epsilon_{L} V_{2}^{2}-\chi_{0}\right) N_{4}+N_{3} \chi_{0}}{2 w^{2}} .
$$

Anyway, the modification of the attenuation coefficients, due to $N_{i}$, will be small because $w^{2}-$ $V_{2}^{2}$ is small and the coefficients $N_{i}$ are also small in the considered situation. This is in contrast with what happens at low frequency, or when the vortex tangle is assumed as perfectly rigid, not affected by the second sound, in which case the relative motion of the normal fluid with respect to the vortex lines yields an attenuation which allows to determine the vortex line density $L$ of the tangle. However, the wave character of vortex density perturbations at high frequency makes that vortex lines and the second sound become two simultaneous waves with a low joint dissipation, in the first-order approach. Thus, from the practical point of view, it seems that, at high frequency, second sound will not provide much information on the vortex tangle because the influence of the average vortex line density $L$ is small both in the speed as in the attenuation. In the next section we will propose an extended hydrodynamical model which includes flux of vortices as independent variable in order to study vortex density waves.

\section{The flux of line density $L$ as new independent variable, vortex density waves}

The vortex lines and their evolution are investigated by second sound waves, so that it is necessary to analyze in depth their mutual interactions. In particular, high-frequency second 
sound may be of special interest to probe small length scales in the tangle, which is necessary in order to explore, for instance, the statistical properties of the vortex loops of several sizes. In fact, the reduction of the size of space averaging is one of the active frontiers in second sound techniques applied to turbulence, but at high-frequencies, the response of the tangle to the second sound is expected to be qualitatively different than at low frequencies, as its perturbations may change from diffusive to propagative behavior (Mongiovì \& Jou, 2007), (Nemirovskii \& Lebedev, 1983), (Yamada et al, 1989), (Jou et al., 2007).

In Section 4 and in the paper (Mongiovì \& Jou, 2007) a thermodynamical model of inhomogeneous superfluid turbulence was built up with the fundamental fields: density $\rho$, velocity $\mathbf{v}$, internal energy density $E$, heat flux $\mathbf{q}$ and average vortex line length per unit volume L. In (Jou et al., 2007), starting from this model, a semiquantitative expression for the vortex diffusion coefficient was obtained and the interaction between second sound and the tangle in the high-frequency regime was studied. In both these works the diffusion flux of vortices $\mathbf{J}$ was considered as a dependent variable, collinear with the heat flux $\mathbf{q}$, which is proportional to the counterflow velocity $\mathbf{V}_{n s}$. But, in general, this feature is not strictly verified because the vortices move with a velocity $\mathbf{v}_{L}$, which is not collinear with the counterflow velocity (for more detail see paper (Sciacca et al, 2008)).

\subsection{Balance equations and constitutive theory}

In this section we build up a thermodynamical model of inhomogeneous counterflow superfluid turbulence, which chooses as fundamental fields the energy density $E$, the heat flux $\mathbf{q}$, the averaged vortex line length per unit volume $L$, and the vortex diffusion flux $\mathbf{J}$. Because experiments in counterflow superfluid turbulence in the linear regime are characterized by a zero value of the barycentric velocity $\mathbf{v}$, in this paper one does not consider $\mathbf{v}$ as independent variable. In a more complete model $\mathbf{v}$ and $\rho$ will be also fundamental fields.

Consider the following balance equations

$$
\left\{\begin{array}{l}
\frac{\partial E}{\partial t}+\frac{\partial q_{k}}{\partial x_{k}}=0 \\
\frac{\partial q_{i}}{\partial t}+\frac{\partial J_{i k}^{q}}{\partial x_{k}}=\sigma_{i}^{q} \\
\frac{\partial L}{\partial t}+\frac{\partial J_{k}}{\partial x_{k}}=\sigma^{L} \\
\frac{\partial J_{i}}{\partial t}+\frac{\partial F_{i k}}{\partial x_{k}}=\sigma_{i}^{\mathbf{J}}
\end{array}\right.
$$

where $E$ is the specific energy per unit volume of the superfluid component plus the normal component plus the vortex lines, $J_{i j}^{q}$ the flux of the heat flux, $J_{i}$ the flux of vortex lines (which was denoted with $J_{i}^{L}$ in the previous Section), and $F_{i j}$ the flux of the flux of vortex lines; $\sigma_{i}^{q}$, $\sigma_{L}$ and $\sigma_{i}^{\mathbf{J}}$ are the respective production terms. Since here one is interested to study the linear propagation of the second sound and vortex density waves, the convective terms have been neglected.

If one supposes that the fluid is isotropic, the constitutive equations for the fluxes $J_{i j}^{q}$ and $F_{i j}$, to the first order in $q_{i}$ and $J_{i}$, can be expressed in the form

$$
\begin{aligned}
& J_{i k}^{q}=\beta_{1}(E, L) \delta_{i k} \\
& F_{i k}=\psi_{1}(E, L) \delta_{i k} .
\end{aligned}
$$


Restrictions on these relations are obtained, as in Section 4, imposing the validity of the second law of thermodynamics, applying Liu's procedure.

In order to make the theory internally consistent, one must consider for entropy density $S$ and entropy flux density $J_{k}^{S}$ approximate constitutive relations to second order in $q_{i}$ and $J_{i}$

$$
S=S_{0}(E, L)+S_{1}(E, L) q^{2}+S_{2}(E, L) J^{2}+S_{3}(E, L) q_{i} J_{i}, \quad J_{k}^{S}=\phi^{q}(E, L) q_{k}+\phi^{J}(E, L) J_{k} .
$$

The quantities $\Lambda^{E}, \Lambda_{i}^{q}, \Lambda^{L}$ and $\Lambda_{i}^{J}$ are Lagrange multipliers, which are also objective functions of $E, q_{i}, L$ and $J_{i}$; in particular, one sets

$$
\begin{gathered}
\Lambda^{E}=\Lambda^{E}\left(E, L, q_{i}, J_{i}\right)=\Lambda_{0}^{E}(E, L)+\Lambda_{1}^{E}(E, L) q^{2}+\Lambda_{2}^{E}(E, L) J^{2}+\Lambda_{3}^{E}(E, L) q_{i} J_{i}, \\
\Lambda^{L}=\Lambda^{L}\left(E, L, q_{i}, J_{i}\right)=\Lambda_{0}^{L}(E, L)+\Lambda_{1}^{L}(E, L) q^{2}+\Lambda_{2}^{L}(E, L) J^{2}+\Lambda_{3}^{L}(E, L) q_{i} J_{i}, \\
\Lambda_{i}^{q}=\lambda_{11} q_{i}+\lambda_{12} J_{i} \quad \text { and } \Lambda_{i}^{J}=\lambda_{21} q_{i}+\lambda_{22} J_{i},
\end{gathered}
$$

with $\lambda_{m n}=\lambda_{m n}(E, L)$. Imposing that the coefficients of the time derivatives are zero, one obtains

$$
d S=\Lambda^{E} d E+\Lambda_{i}^{q} d q_{i}+\Lambda^{L} d L+\Lambda_{i}^{J} d J_{i}
$$

In the same way, imposing that the coefficients of space derivatives vanish, one finds

$$
d J_{k}^{S}=\Lambda^{E} d q_{k}+\Lambda_{i}^{q} d J_{i k}^{q}+\Lambda^{L} d J_{k}+\Lambda_{i}^{J} d F_{i k} .
$$

Substituting now (126), (127) and (128) in (129-130), one gets

$$
\begin{gathered}
S_{1}=\frac{1}{2} \lambda_{11}, \quad S_{2}=\frac{1}{2} \lambda_{22}, \quad S_{3}=\lambda_{12}=\lambda_{21}, \\
\phi^{q}=\Lambda_{0}^{E}, \quad \phi^{J}=\Lambda_{0}^{L}, \\
d S_{0}=\Lambda_{0}^{E} d E+\Lambda_{0}^{L} d L, \quad d S_{1}=\Lambda_{1}^{E} d E+\Lambda_{1}^{L} d L, \\
d S_{2}=\Lambda_{2}^{E} d E+\Lambda_{2}^{L} d L, \quad d S_{3}=\Lambda_{3}^{E} d E+\Lambda_{3}^{L} d L, \\
d \phi^{q}=\lambda_{11} d \beta_{1}+\lambda_{21} d \psi_{1}, \quad d \phi^{J}=\lambda_{12} d \beta_{1}+\lambda_{22} d \psi_{1} .
\end{gathered}
$$

In particular, one obtains to the second order in $\mathbf{q}$ and $\mathbf{J}$ the following expressions for the entropy and for the entropy flux

$$
S=S_{0}+\frac{1}{2} \lambda_{11} q^{2}+\frac{1}{2} \lambda_{22} J^{2}+\lambda_{12} q_{i} J_{i}, \quad J_{k}^{S}=\Lambda_{0}^{E} q_{k}+\Lambda_{0}^{L} J_{k}
$$

It remains the following residual inequality for the entropy production

$$
\sigma^{S}=\Lambda_{i}^{q} \sigma_{i}^{q}+\Lambda^{L} \sigma_{L}+\Lambda_{i}^{J} \sigma_{i}^{J} \geq 0
$$

Now, the obtained relations are analyzed in detail. As in Section 4 we first introduce a generalized temperature as the reciprocal of the first-order part of the Lagrange multiplier of the energy: 


$$
\Lambda_{0}^{E}=\left[\frac{\partial S_{0}}{\partial E}\right]_{L}=\frac{1}{T}
$$

and the chemical potential of vortex lines (near equilibrium):

$$
-T \Lambda_{0}^{L}=\mu^{L} .
$$

Neglecting in (129) second order terms in $\mathbf{q}$ and $\mathbf{J}$, and using relations (131), (138) and (139), the following expression for the entropy density $S$ is obtained

$$
d S=\frac{1}{T} d E-\frac{\mu^{L}}{T} d L+\lambda_{11} q_{i} d q_{i}+\lambda_{22} J_{i} d J_{i}+\lambda_{12}\left(J_{i} d q_{i}+q_{i} d J_{i}\right)
$$

Consider now equations (135), which one rewrites using (132) and (139) as

$$
d\left(\frac{1}{T}\right)=\lambda_{11} d \beta_{1}+\lambda_{21} d \psi_{1}, \quad d\left(-\frac{\mu^{L}}{T}\right)=\lambda_{12} d \beta_{1}+\lambda_{22} d \psi_{1} .
$$

After some calculations (Sciacca et al, 2008), we find:

$$
\begin{aligned}
& \zeta_{1}=\frac{\partial \beta_{1}}{\partial T}=\frac{1}{N}\left[-\frac{1}{T^{2}} \lambda_{22}+\lambda_{12} \frac{\partial}{\partial T}\left(\frac{\mu_{0}^{L}}{T}\right)\right], \quad \chi_{1}=\frac{\partial \beta_{1}}{\partial L}=\frac{1}{T} \frac{\lambda_{12}}{N} \frac{\partial \mu_{0}^{L}}{\partial L} \\
& \eta_{1}=\frac{\partial \psi_{1}}{\partial T}=\frac{1}{N}\left[\frac{1}{T^{2}} \lambda_{12}-\lambda_{11} \frac{\partial}{\partial T}\left(\frac{\mu_{0}^{L}}{T}\right)\right], \quad v_{1}=\frac{\partial \psi_{1}}{\partial L}=-\frac{1}{T} \frac{\lambda_{11}}{N} \frac{\partial \mu_{0}^{L}}{\partial L}
\end{aligned}
$$

where $N=\lambda_{11} \lambda_{22}-\lambda_{12}{ }^{2}$. A physical meaning for the coefficient $\lambda_{22}$ was furnished in (Sciacca et al, 2008). Finally, one obtains for the entropy flux

$$
J_{k}^{S}=\frac{1}{T} q_{k}-\frac{\mu_{0}^{L}}{T} J_{k}
$$

which is analogous to the usual expression of the entropy flux in the presence of a mass flux and heat flux, but with the second term related to vortex transport rather than to mass transport.

Substituting the constitutive equations (126) in system (125), using the relations (142-143), and expressing the energy $E$ in terms of $T$ and $L$, the following system of field equations is obtained

$$
\left\{\begin{array}{l}
\rho c_{V} \frac{\partial T}{\partial t}+\rho \epsilon_{L} \frac{\partial L}{\partial t}+\frac{\partial q_{j}}{\partial x_{j}}=0 \\
\frac{\partial q_{i}}{\partial t}+\zeta_{1} \frac{\partial T}{\partial x_{i}}+\chi_{1} \frac{\partial L}{\partial x_{i}}=\sigma_{i}^{q} \\
\frac{\partial L}{\partial t}+\frac{\partial J_{j}}{\partial x_{j}}=\sigma^{L} \\
\frac{\partial J_{i}}{\partial t}+\eta_{1} \frac{\partial T}{\partial x_{i}}+v_{1} \frac{\partial L}{\partial x_{i}}=\sigma^{J_{i}}
\end{array}\right.
$$

where $c_{V}$ is the specific heat at constant volume and $\epsilon_{L}=\partial E / \partial L$. The coefficients $\chi_{1}$ and $\eta_{1}$ describe cross effects linking the dynamics of $\mathbf{q}$ and $\mathbf{J}$ with $L$ and $T$, respectively. Thus, they are expected to settle an interaction between heat waves and vortex density waves. These 
equations are analogous to those proposed in Section 4 (Mongiovì \& Jou, 2007) except for the choice of $J_{i}$ : in fact here $J_{i}$ is assumed to be an independent field whereas in Section 4 (Mongiovì \& Jou, 2007) $J_{i}$ was assumed as dependent on $q_{i}$. However, at high frequency, $J_{i}$ will become dominant and will play a relevant role, as shown in the following.

The production terms $\sigma$ must also be specified. Regarding $\sigma_{i}^{q}$ and $\sigma^{L}$, since only counterflow situation is considering, as in Section 4, we assume

$$
\sigma^{\mathbf{q}}=-K_{1} L \mathbf{q}, \quad \sigma^{L}=-\beta_{q} L^{2}+\alpha_{q}|q| L^{3 / 2},
$$

where $K_{1}=\frac{1}{3} \kappa B$. For the production term of vortex line diffusion, one assumes the following relaxational expression:

$$
\vec{\sigma}^{J}=-\frac{\mathbf{J}}{\tau_{J}}=-\gamma_{1} \kappa L \mathbf{J},
$$

where $\gamma_{1}$ is a positive coefficient which can depend on the temperature $T$ (Sciacca et al, 2008). Note that in (146) one has assumed that the production terms of $\mathbf{q}$ and $\mathbf{J}$ depend on $\mathbf{q}$ and $\mathbf{J}$, respectively, but not on both variables. In more general terms, one could assume that both production terms depend on the two fields $\mathbf{q}$ and $\mathbf{J}$ simultaneously.

In order to determine the physical meaning of the coefficients appearing in equations (145)-(147), concentrate first the attention on the equations for $L$ and J. Supposing that J varies very slowly, one obtains (Sciacca et al, 2008)

$$
\frac{\partial}{\partial t} L=\frac{\eta_{1}}{\gamma_{1} \kappa L} \nabla^{2} T+\frac{v_{1}}{\gamma_{1} \kappa L} \nabla^{2} L+\sigma^{L} .
$$

It is then seen that the coefficient $\frac{v_{1}}{\gamma_{1} \kappa L} \equiv \bar{D}_{1}$ represents the diffusion coefficient of vortices. Coefficient $\frac{\eta_{1}}{\gamma_{1} \kappa L} \equiv \bar{D}_{2}$ may be interpreted as a thermodiffusion coefficient of vortices because it links the temperature gradient to vortex diffusion. In other terms, this implies a drift of the vortex tangle. Detailed measurements have indeed shown [(Donnelly, 1991), pag.216] a slow drift of the tangle towards the heater; this indicates that $\eta_{1}<0$ and small. The hypothesis $\eta_{1}=0$ corresponds to $\bar{D}_{2}=0$, i.e. the vortices do not diffuse in response to a temperature gradient.

\subsection{Interaction of second sound and vortex density waves}

In this Section wave propagation in counterflow vortex tangles is studied, with the aim to discuss the physical effects of the interaction between high-frequency second sound and vortex density waves. A stationary solution of the system (145), with the expressions of the production terms (146-147), is

$$
\begin{gathered}
\mathbf{q}=\mathbf{q}_{0}=\left(q_{01}, 0,0\right), \quad L=L_{0}=\frac{\alpha_{q}^{2}}{\beta_{q}^{2}} q_{01}^{2}, \\
T=T_{0}(\mathbf{x})=T^{*}-\frac{1}{3} \frac{\kappa B}{\zeta_{1}} L_{0} q_{01} x_{1}, \quad \mathbf{J}_{0}=\left(\frac{1}{3} \frac{\kappa B}{\zeta_{1} \gamma_{1} \kappa} q_{01}, 0,0\right),
\end{gathered}
$$

with $q_{01}>0$.

Consider the propagation of harmonic plane waves of the four fields of the equation (145) in the following form 


$$
\left\{\begin{array}{l}
T=T_{0}(\mathbf{x})+\tilde{T} e^{i(K \mathbf{n} \cdot \mathbf{x}-\omega t)} \\
\mathbf{q}=\mathbf{q}_{0}+\tilde{\mathbf{q}} e^{i(K \mathbf{n} \cdot \mathbf{x}-\omega t)} \\
L=L_{0}+\tilde{L} e^{i(K \mathbf{n} \cdot \mathbf{x}-\omega t)} \\
\mathbf{J}=\mathbf{J}_{0}+\tilde{\mathbf{J}} e^{i(K \mathbf{n} \cdot \mathbf{x}-\omega t)}
\end{array}\right.
$$

where $K=k_{r}+i k_{s}$ is the wave number, $\omega$ the real frequency, $\mathbf{n}$ the unit vector along the direction of the wave propagation.

Substituting (151) in the system (145), and linearizing the quantities (146), and (147) around the stationary solutions, the following equations for the small amplitudes are obtained

$$
\left\{\begin{array}{l}
-\omega\left[\rho c_{V}\right]_{0} \tilde{T}-\omega\left[\rho \epsilon_{L}\right]_{0} \tilde{L}+K \tilde{\mathbf{q}} \cdot \mathbf{n}=0 \\
{\left[-\omega-\frac{i}{3} \kappa B L_{0}\right] \tilde{\mathbf{q}}+\zeta_{10} K \tilde{T} \mathbf{n}-\left(-\chi_{10} K \mathbf{n}+\frac{i}{3} \kappa B \mathbf{q}_{0}\right) \tilde{L}=0} \\
{\left[-\omega-i\left(2 \beta_{q} L_{0}-\frac{3}{2} \alpha_{q} L_{0}^{1 / 2} q_{01}\right)\right] \tilde{L}+K \tilde{\mathbf{J}} \cdot \mathbf{n}+i \alpha_{q} L_{0}^{3 / 2} \tilde{q_{1}}=0} \\
\left(-\omega-i \gamma_{1} \kappa L_{0}\right) \tilde{\mathbf{J}}+\eta_{10} K \mathbf{n} \tilde{T}+\left(\nu_{10} K \mathbf{n}-i \gamma_{1} \kappa \mathbf{J}_{0}\right) \tilde{L}=0
\end{array}\right.
$$

Note that the subscript 0 refers to the unperturbed state; in what follows, this subscript will be dropped out to simplify the notation.

First case: $\mathbf{n}$ parallel to $\mathbf{q}_{\mathbf{0}}$.

Now, impose the condition that the direction of the wave propagation $\mathbf{n}$ is parallel to the heat flux $\mathbf{q}_{\mathbf{0}}$, namely $\mathbf{n}=(1,0,0)$. Through these conditions the system (152) becomes

$$
\left\{\begin{array}{l}
-\omega \rho c_{V} \tilde{T}+K \tilde{q}_{1}-\omega \rho \epsilon_{L} \tilde{L}=0 \\
\zeta_{1} K \tilde{T}-\left(\omega+\frac{i}{3} \kappa B L\right) \tilde{q}_{1}-\left(-\chi_{1} K+i \kappa \frac{B}{3} q_{1}\right) \tilde{L}=0 \\
i \alpha_{q} L^{3 / 2} \tilde{q}_{1}-\left(\omega+i \tau_{L}^{-1}\right) \tilde{L}+K \tilde{J}_{1}=0 \\
\eta_{1} K \tilde{T}+\left(\nu_{1} K-i \gamma_{1} \kappa J_{1}\right) \tilde{L}+\left(-\omega-i \gamma_{1} \kappa L\right) \tilde{J}_{1}=0 \\
\left(-\omega-\frac{i}{3} \kappa B L\right) \tilde{q}_{2}=0 \\
\left(-\omega-\frac{i}{3} \kappa B L\right) \tilde{q}_{3}=0 \\
\left(-\omega-i \gamma_{1} \kappa L\right) \tilde{J}_{2}=0 \\
\left(-\omega-i \gamma_{1} \kappa L\right) \tilde{J}_{3}=0
\end{array}\right.
$$

where $\tau_{L}^{-1}=\left(2 \beta_{q} L-\frac{3}{2} \alpha_{q} L^{1 / 2} q_{1}\right)$.

Note that the transversal modes, those corresponding to the four latter equations, evolve independently with respect to the longitudinal ones, corresponding to the four former equations.

One will limit the study to the case in which $\omega$ and the modulus of the wave number $K$ assume values high enough to make considerable simplification in the system. Indeed, it is for high values of the frequency that the wave behavior of the vortex tangle can be evidenced because the first term in (145c) will become relevant. Note that the assumption $|K|=\left|k_{r}+i k_{s}\right|$ large 


\begin{tabular}{|l|l|}
\hline$w_{1,2}= \pm V_{2}$ & $w_{3,4}= \pm \sqrt{v_{1}}$ \\
\hline \hline & \\
$\tilde{T}=\psi$ & $\tilde{T}=-\frac{1}{\rho c_{V}}\left(\frac{\chi_{1}-v_{1} \rho \epsilon_{L}}{V_{2}^{2}-v_{1}}\right) \psi$ \\
$\tilde{q}_{1}= \pm V_{2} \rho c_{V} \psi$ & $\tilde{q}_{1}= \pm \frac{\sqrt{v_{1}}\left(\rho \epsilon_{L} V_{2}^{2}-\chi_{1}\right)}{V_{2}^{2}-v_{1}} \psi$ \\
$\tilde{L}=0$ & $\tilde{L}=\psi$ \\
$\tilde{J}_{1}=0$ & $\tilde{J}_{1}= \pm \sqrt{v_{1}} \psi$ \\
\hline
\end{tabular}

Table 1. Modes corresponding to second sound velocity and vortex density waves, respectively.

refers to a large value of its real part $k_{r}$, which is related to the speed of the vortex density wave, whereas the imaginary part $k_{s}$, corresponding to the attenuation factor of the wave, will be assumed small.

This problem is studied into two steps: first assuming $|K|$ and $\omega$ extremely high to neglect all terms which do not depend on them. Then, the solution so obtained is perturbed in order to evaluate the influence of the neglected terms on the velocity and the attenuation of high-frequency waves.

Denoting with $w=\omega / k_{r}$ the speed of the wave, and assuming $|K|$ and $\omega$ large, the following dispersion relation is obtained:

$$
w^{4}-\left[V_{2}^{2}+v_{1}-\frac{\eta_{1}}{\rho c_{V}}\left(\rho \epsilon_{L}-\frac{\chi_{1}}{v_{1}}\right)\right] w^{2}+V_{2}^{2} v_{1}=0,
$$

where $V_{2}=\left(-\lambda_{22} T^{2} \rho c_{V}\right)^{-1 / 2}$ is the second sound speed in the absence of vortex tangle (see previous sections) and from (142b) it is related to the coefficient $\zeta_{1}$ by the relation $\zeta_{1}=V_{2}^{2} \rho c_{V}-$ $\lambda_{12} \eta_{1} / \lambda_{11}$. Further, if one assumes that the coefficient $\eta_{1}$ is zero

$$
\eta_{1}=0 \quad \Rightarrow \quad \frac{\lambda_{12}}{\lambda_{11}}=T^{2} \frac{\partial}{\partial T}\left(\frac{\mu^{L}}{T}\right)=\frac{2 S_{3}}{S_{2}}=-\frac{\chi_{1}}{v_{1}},
$$

then the dispersion relation (154) has the solutions

$$
w_{1,2}= \pm V_{2}, \quad w_{3,4}= \pm \sqrt{v_{1}}
$$

to which correspond the propagation modes shown in Table 1.

As one sees from the first column of Table 1, under the hypothesis (155) the high-frequency wave of velocity $w_{1,2}= \pm V_{2}$ is a temperature wave (i.e. the second sound) in which the two quantities $\tilde{L}$ and $\tilde{J}_{1}$ are zero, whereas in the second column the high-frequency wave of velocity $w_{3,4}= \pm \sqrt{v_{1}}$ is a wave in which all fields vibrate. The latter result is logic because when the vortex density wave is propagated in the superfluid helium, temperature $T$ and heat flux $q_{1}$ cannot remain constant. This behavior is different from that obtained in Section 4 , because using that model in the second sound also the line density $L$ vibrates. In fact, there the flux of vortices $\mathbf{J}$ was chosen proportional to $\mathbf{q}$, so that vibrations in the heat flux (second sound) produce vibrations in the vortex tangle. Experiments on high-frequency second sound are needed to confirm this new result.

Now we consider all the neglected terms of the system (153) and the coefficient $\eta_{1}$ as small perturbations of the velocity $w$ of the wave and of the attenuation term $k_{s}$ of the wave number $K$. Substituting the following assumptions 


$$
\bar{w}=\frac{\omega}{k_{r}}=w+\delta \quad \text { and } \quad K=k_{r}+i k_{s}
$$

in the system (153), one find the expression (156), at the zeroth order in $\delta$ and $k_{s}$, whereas at the first order in $\delta$ and $k_{s}$, one obtains

$$
\begin{aligned}
& \bar{w}_{1,2}^{\|}=\left(1-\frac{\eta_{1}}{2 \rho c_{V}\left(w_{1,2}^{2}-w_{3,4}^{2}\right)}\left(\rho \epsilon_{L}-\frac{\chi_{1}}{w_{3,4}^{2}}\right)\right) w_{1,2}, \\
& \bar{w}_{3,4}^{\|}=\left(1+\frac{\eta_{1}}{2 \rho c_{V}\left(w_{1,2}^{2}-w_{3,4}^{2}\right)}\left(\rho \epsilon_{L}-\frac{\chi_{1}}{w_{3,4}^{2}}\right)\right) w_{3,4},
\end{aligned}
$$

and

$$
\begin{gathered}
k_{s}^{(1,2)}=\frac{\kappa L B}{6 w_{1,2}}+\frac{\alpha_{q} L^{3 / 2}\left(w_{1,2}^{2} \rho \epsilon_{L}-\chi_{1}\right)}{2\left(w_{1,2}^{2}-w_{3,4}^{2}\right)}, \\
k_{s}^{(3,4)}=\frac{\kappa L \gamma_{1}+\tau_{L}^{-1}}{2 w_{3,4}}-\frac{\alpha_{q} L^{3 / 2}\left(w_{1,2}^{2} \rho \epsilon_{L}-\chi_{1}\right)}{2\left(w_{1,2}^{2}-w_{3,4}^{2}\right)}+\frac{J_{1} \kappa \gamma_{1}}{2 w_{3,4}^{2}} .
\end{gathered}
$$

Observe that in this approximation all thermodynamical fields vibrate simultaneously and the attenuation coefficients $k_{s}$ are influenced by the choice of $\mathbf{J}$ as independent variable, as one easily sees by comparing expressions (159-160) with those obtained in Section 4 (Jou et al., 2007). Looking at these results, in particular the two speeds (157-158), one sees that these velocities are not modified when one makes the simplified hypothesis that the coefficient $\eta_{1}$ is equal to zero. In Section 4 (Jou et al., 2007) it was observed that the second sound velocity is much higher than that of the vortex density waves, so that the small quantity $\eta_{1}$ should influence the two velocities (157-158) in a different way: negligible for the second sound velocity but relevant for the vortex density waves. Regarding the attenuation coefficients (159-160), one sees that the first term in (159) is identical to that obtained in (Jou et al., 2002), when the vortices are considered fixed. The new term, proportional to $\alpha_{q}$, comes from the interaction between second sound and vortex density waves.

Note that the second term of the dissipative coefficient $k_{s}^{(1,2)}$ is the same as the third term of $k_{s}^{(3,4)}$, but with an opposite sign. This means that this term contributes to the attenuation of the two waves in opposite ways; and its contribution depends also on whether the propagation of forward waves or of backward waves is considered. The first term of $k_{s}^{(3,4)}$ produces always an attenuation of the wave, while the behavior of the third term is analogous to the first one.

Second case: $\mathbf{n}$ orthogonal to $\mathbf{q}_{\mathbf{0}}$

In order to make a more detailed comparison with the model studied in Section 4 (Mongiovì \& Jou, 2007), (Jou et al., 2007), one proceeds to analyze another situation, in which the direction of the wave propagation is perpendicular to the heat flux, that is, for example, assuming $\mathbf{n}=(0,0,1)$. This choice simplifies the system (152) in the following form 


$$
\left\{\begin{array}{l}
-\omega \rho c_{V} \tilde{T}+K \tilde{q}_{3}-\omega \rho \epsilon_{L} \tilde{L}=0 \\
\left(-\omega-\frac{i}{3} \kappa B L\right) \tilde{q}_{1}-\frac{i}{3} \kappa B q_{1} \tilde{L}=0 \\
\zeta_{1} K \tilde{T}-\left(\omega+\frac{i}{3} \kappa B L\right) \tilde{q}_{3}+\chi_{1} K \tilde{L}=0 \\
i \alpha_{q} L^{3 / 2} \tilde{q}_{1}-\left(\omega+i \tau_{L}^{-1}\right) \tilde{L}+K \tilde{J}_{3}=0 \\
\eta_{1} K \tilde{T}+v_{1} K \tilde{L}+\left(-\omega-i \gamma_{1} \kappa L\right) \tilde{J}_{3}=0 \\
\left(-\omega-\frac{i}{3} \kappa B L\right) \tilde{q}_{2}=0 \\
-i \gamma_{1} \kappa J_{1} \tilde{L}+\left(-\omega-i \gamma_{1} \kappa L\right) \tilde{J}_{1}=0 \\
\left(-\omega-i \gamma_{1} \kappa L\right) \tilde{J}_{2}=0
\end{array}\right.
$$

Note that, in contrast with what was seen before, but in agreement with the corresponding situation of the model described in Section 4, here the transversal and the longitudinal modes in general do not evolve independently, as shown from the first five equations. However, one will see that this is the case if high-frequency waves are considered.

As in the previous situation, we assume that the values of the frequencies $\omega$ and of the real part of the wave number, $k_{r}$, are high enough, such that the system (161) may be easily solved. The other terms will be considered as perturbations to $w=\omega / k_{r}$ and $k_{s}$. Note that in this special case, as in the previous case and in (Jou et al., 2007), only the longitudinal modes are present, so that the dispersion relation assumes the form

$$
w\left(w^{4}-\left[V_{2}^{2}+v_{1}-\frac{\eta_{1}}{\rho c_{V}}\left(\rho \epsilon_{L}+\frac{\lambda_{12}}{\lambda_{11}}\right)\right] w^{2}+V_{2}^{2} v_{1}\right)=0,
$$

which is similar to equation (154).

Assuming the same hypothesis (155), the dispersion relation (162) takes the form

$$
w\left(w^{2}-v_{1}\right)\left(w^{2}-V_{2}^{2}\right)=0,
$$

where $V_{2}$ is the second sound velocity and $\sqrt{v_{1}}$ is the velocity of the vortex density waves in helium II. The conclusions which one achieves here are the same to those of the previous situation. Indeed, $\omega_{0}=0$ corresponds to $\tilde{q}_{1}=\psi$ and $\tilde{T}=\tilde{q}_{3}=\tilde{L}=\tilde{J}_{3}=0$; while $w_{1,2}= \pm V_{2}$ and $w_{3,4}= \pm \sqrt{v_{1}}$ correspond to those in Table 1 .

Now, as in the previous case, we assume that all the neglected terms in (161) modify $w$ and $K$ by small quantities $\delta$ and $k_{s}$, that is

$$
\bar{w}=\frac{\omega}{k_{r}}=w+\delta \quad \text { and } \quad K=k_{r}+i k_{s} .
$$

Substituting them in the dispersion relation of the system (161), one finds the relation (163), at the zeroth order in $\delta$ and $k_{s}$, and the following two expressions at the first order in $\delta$ and $k_{s}$

and

$$
\begin{aligned}
& \bar{w}_{1,2}^{\perp}=\bar{w}_{1,2^{\prime}}^{\|} \\
& \overline{w_{3,4}} \stackrel{\perp}{=}=\bar{w}_{3,4^{\prime}}^{\|}
\end{aligned}
$$

$$
k_{s}^{(1,2)}=\frac{\kappa L B}{6 w_{1,2}}
$$




$$
k_{s}^{(3,4)}=\frac{\tau_{L}^{-1}+\kappa L \gamma_{1}}{2 w_{3,4}} .
$$

As regards the expression (166) for the dissipative term $k_{S}^{(1,2)}$, note that it is the same as the expression obtained when the vortices are assumed fixed (Jou \& Mongiovì, 2006), (Peruzza \& Sciacca, 2007), whereas the attenuation term $k_{s}^{(3,4)}$ is the same as the second term of $k_{s}^{(3,4)}$ of the first case (n parallel to $\mathbf{q}_{0}$ ). As in (Mongiovì \& Jou, 2007), (Jou et al., 2007), in this case one has the propagation of two kinds of waves, namely heat waves and vortex density waves, which cannot be considered as propagating independently from each other. In fact, the uncoupled situation (equation (156)), in which the propagation of the second sound is not influenced by the fluctuations of the vortices, is no more the case when the quantities $\bar{N}_{1}=\frac{1}{3} \kappa B L, \bar{N}_{2}=\frac{1}{3} \kappa B q_{1}, \bar{N}_{3}=A L^{3 / 2}, \bar{N}_{4}=\gamma_{1} \kappa J_{1}, \bar{N}_{5}=\gamma_{1} \kappa L, \tau_{L}^{-1}$ and $\eta_{1}$, are considered. Indeed, from (157-158) and from the results of (Jou et al., 2007) one makes in evidence that heat and vortex density waves cannot be considered separately, that is as two different waves, but as two different features of the same phenomena. Of course, the results obtained here are more exhaustive than those of Section 4: in fact, comparing the velocities at the first order of approximation in both models, one deduces that the expressions (157-158) depend not only on the velocities of heat waves and vortex density waves, as in (Mongiovì \& Jou, 2007), (Jou et al., 2007), but also on the coefficient $\eta_{1}$, which comes from the equation (145d) of the vortex flux $\mathbf{J}$, and whose physical meaning is a thermodiffusion coefficient of vortices. The fourth equation of the system (153) shows that the vortex flux $\tilde{J}_{1}$ is not proportional to the heat flux, as it was assumed in Sections 2 and 4, but it satisfies an equation in which also the fields $\tilde{L}$ and $\tilde{T}$, through $\eta_{1}$, are present.

It is to note that the attenuation of the second sound depends on the relative direction of the wave with respect to the heat flux: in some experiments this dependence was shown for parallel and orthogonal directions (Awschalom et al, 1984). These results were explained assuming an anisotropy of the tangle of vortices. But, looking at the expressions (159) and (166) of the attenuation of the second sound in the high-frequency regime, one notes that these expressions are not equal. In particular, the term

$$
\frac{\alpha_{q} L^{3 / 2}\left(w_{1,2}^{2} \rho \epsilon_{L}-\chi_{1}\right)}{2\left(w_{1,2}^{2}-w_{3,4}^{2}\right)}
$$

in (159) causes a dependence of the attenuation depending on whether the wave direction agrees with the direction of the heat flux $\mathbf{q}$ or not. This term is absent if the wave propagates orthogonal to the heat flux. In (Sciacca et al, 2008) vortex tangle was assumed to be anysotropic. The result was that $\bar{w}_{1,2,3,4}^{\perp}=\bar{w}_{1,2,3,4}^{\|}$and that the behavior of speed of propagation is isotropic and does not depend on the isotropy or anisotropy of the tangle. In conclusion, it could be that an anisotropy of the behavior of high-frequency second sound does not necessarily imply an actual anisotropy of the tangle in pure counterflow regime, but only a different behavior of the second sound due to the interaction with the vortex density waves. This may be of interest if one wants to explore the degree of isotropy at small spatial scales. Of course, some more experiments are needed in order to establish the presence and the sign of these additional terms. 


\section{Conclusions and perspectives}

Helium behaves in a very strange way when temperature is dropped down below the lambda line, different to any classical fluid. This review is a first attempt to put together some of our results concerning the application of the Extended Thermodynamics to superfluid helium, both in laminar and turbulent flows.

In Section 2 a one-fluid model for superfluid helium in absence of vortices is shown, which chooses heat flux as an independent variable, and a comparison between this non-standard model and the more well-known two-fluid model is faced. The main part of the review is devoted to the macroscopic description of the interesting behaviour of this liquid in the presence of quantized vortex lines. They are very thin dynamical defects of superfluid helium, which are usually sketched by geometrical lines, representing the quantized vorticity of the superfluid motion. The amount of quantized vortices is high enough in turbulent superfluid helium, so they are usually expressed by means of the line length per unit volume $L$. Different hydrodynamical models of superfluids in the presence of vortices are dealt with, that have more detailed successive descriptions. First, in Section 3 the one-fluid model for laminar flow (no presence of vortices) is extended introducing a vorticity tensor (in the heat flux equation), which takes into account the presence of vortices as a fixed structure. The influence of vortices to the main fields is studied, mainly in the three experimental situations: rotating helium (vortices are basically straight lines parallel to the rotating axis), pure counterflow (an enough high heat flux, without mass flux, which causes an almost isotropic vortex tangle), and then the combined situation of rotating counterflow turbulence.

Since vortex lines density may experimentally be detected by means of the second sound (temperature waves), the propagation of harmonic waves is investigated in all the situations above mentioned. Section 4 is devoted to build up a new model in which the line density $L$ acquires field properties: it depends on the coordinates, it has a drift velocity, and it has associated a diffusion flux. These features are becoming increasingly relevant today, as the local vortex density may be measured with higher precision, and the relative motion of vortices is observed and simulated. The hydrodynamical model built in this section is sufficiently general to encompass vortex diffusion and to describe the interactions between the usual waves and the vortices, which in Section 3 were simply considered as a rigid framework where second sound waves are dissipated. A hint about vortex density waves is also shown, which is then better considered in Section 5. In this section we further generalize the model, in order to include the velocity of the vortex tangle as a new independent variable. This is motivated by the fact that this velocity (or the flux of the vortex line density) is not always properly parallel to the heat flux, so it needs an own evolution equation. Also this model is formulated using Extended Thermodynamics, determining the restrictions imposed by the second law of Thermodynamics by means of the Liu's procedure. One of the results of this section is that when the high frequency harmonic plane waves are considered, vortex density waves are found out. The interesting thing is that heat waves and vortex density waves cannot be considered separately, that is as two different waves, but as two different features of the same phenomena. Another interesting result is that attenuation of the second sound depends on the relative direction of the wave with respect to the heat flux: it seems that the anisotropy in the behavior of high-frequency second sound does not mean anisotropy in the tangle in counterflow regime.

These results are important because second sound provides the standard methods of measuring the vortex line density $L$, and we have shown that the dynamical mutual interplay between second sound and vortex lines modifies the standard results. In the case when there 
is a net motion of the mass, the model is useful to study Couette and Poiseuille flow, where the bulk motion of the system contribute to the production of new vortex lines (Jou et al., 2008).

The renewed interest in superfluid turbulence lies on the fact that at some length scales it appears similar to classical hydrodynamic turbulence, and therefore a better understanding of it can throw new light on problems in classical turbulence. Our results are relevant also to modelize the influence of the bulk motion on the vortex production in Couette and Poiseuille flows, and in towed or oscillating grids, including the important application of superfluid helium as a coolant for superconducting devices.

\section{Acknowledgments}

The authors acknowledge the support of the Università di Palermo (grant 2007.ORPA07LXEZ and Progetto CoRI 2007, Azione D, cap. B.U. 9.3.0002.0001.0001) and the collaboration agreement between Università di Palermo and Universitàt Autònoma de Barcelona. MS acknowledges the "Assegno di ricerca" of the University of Palermo. DJ acknowledges the financial support from the Dirección General de Investigación of the Spanish Ministry of Education under grant FIS 2009-13370-C02-01 and of the Direcció General de Recerca of the Generalitat of Catalonia, under grant 2009 SGR-00164.

\section{References}

Ardizzone, L. \& Gaeta, G. (2009). Far from equilibrium constitutive relations in a nonlinear model of Superfluid turbulence, In: Boll. di Mat. Pura ed Appl. Vol. II, Brugarino T., Mongiovì M.S., (Ed.), 59-67, ARACNE, Roma.

Ardizzone, L., Gaeta, G. \& Mongiovì M.S. (2009). A Continuum Theory of Superfluid Turbulence based on Extended Thermodynamics. J. Non-Equilib. Thermodyn., Vol. 34, 277-297.

Awschalom, D.D., Milliken, F.P. \& Schwarz, K.W. (1984). Properties of superfluid turbulence in a large channel. Phys. Rev. Lett., Vol. 53, 1372-1375.

Barenghi, C.F. (1999). Classical aspects of quantum turbulence. J. Phys. Cond. Matter, Vol. 11, 7751-7759.

Barenghi, C.F., Donnelly, R.J. \& Vinen, W.F. (2001). Quantized Vortex Dynamics and Superfluid Turbulence, Springer, Berlin.

Casas-Vázquez, J.\& Jou, D. (2003). Temperature in non-equilibrium states: a review of open problems and current proposals. Rep. Prog. Phys., Vol. 66, 1937-2023.

Donnelly, R. J. \& Swanson, C. E. (1986). Quantum turbulence. J. Fluid Mech., Vol. 173, 387-429.

Donnelly, R.J. (1991). Quantized Vortices in Helium II, Cambridge University Press, Cambridge.

Fazio, R. \& van der Zant, H. (2001). Quantum phase transition and vortex dynamics in superconducting networks. Physics Reports, Vol. 355, 235-334.

Feynman, R.P. (1955). Application of quantum mechanics to liquid helium II, In: Progress in Low Temperature Physics Vol. I, Gorter, C.J., (Ed.), 17-53, North-Holland, Amsterdam.

Gorter, C. J. \& Mellink, J. H. (1949). On the irreversible processes in liquid helium II. Physica, Vol. 15, 285-304.

Hall, H.E. \& Vinen, W.F (1956). The rotation of liquid helium II, I. Experiments the propagation of second sound in uniformly rotating helium II. Proc. Roy. Soc., Vol. A238, 215-234.

Hall, H.E. \& Vinen, W.F (1956). The rotation of liquid helium II, II. The theory of mutual friction in uniformly rotating helium II. Proc. Roy. Soc., Vol. A238, 204-214.

Jou D., Casas-Vázquez, J. \& Lebon, G. (2001). Extended Irreversible Thermodynamics, 
Springer-Verlag, Berlin.

Jou, D., Lebon, G. \& Mongiovì, M.S. (2002). Second sound, superfluid turbulence and intermittent effects in liquid helium II. Phys. Rev. B, Vol. 66, 224509 (9 pages).

Jou, D. \& Mongiovì, M.S. (2004). Phenomenological description of counterflow superfluid turbulence in rotating containers. Phys. Rev. B, Vol. 69, 094513 (7 pages).

Jou, D. \& Mongiovì, M.S. (2005). Non-Equilibrium Thermodynamics in Counterflow and Rotating Situations. Phys. Rev. B, Vol. 72, 144517 (8 pages).

Jou, D. \& Mongiovì, M.S. (2005). Description and evolution of anisotropy in superfluid vortex tangles with counterflow and rotation. Phys. Rev. B, Vol. 74, 054509 (11 pages).

Jou, D., Mongiovì, M.S. \& Sciacca, M. (2007). Vortex density waves and high-frequency second sound in superfluid turbulence hydrodynamics. Phys. Lett. A, Vol. 368, 7-12.

Jou, D., Sciacca, M. \& Mongiovì, M.S. (2008). Vortex dynamics in rotating counterflow and plane Couette and Poiseuille turbulence in superfluid helium, Phys. Rev. B, Vol. 78, 024524 (12 pages).

Khalatnikov, I.M.(1965). An Introduction to the Theory of Superfluidity, Benjamin, New York.

Landau, L.D. (1941). The theory of superfluidity of He II. J. Phys., Vol. 5, 71-90.

Lebon, G., Jou, D. (1979). A continuum theory of liquid helium II based on the classical theory of irreversible processes. J. Non-Equilib.Thermodyn., Vol. 4, 259-276.

Lebon, G., Jou D. \& Casas-Vázquez, J. (2008). Understanding non-equilibrium thermodynamics, Springer-Verlag, Berlin.

Liu,I. (1972). Method of Lagrange multipliers for exploitation of entropy principle. Arch. Rat. Mech. Anal., Vol. 46, 131-148.

Mongiovì, M.S. (1991). Superfluity and entropy conservation in extended thermodynamics. J. Non-Equilib. Thermod., Vol. 16, 225-239.

Mongiovì, M.S. (1993). Extended Irreversible Thermodynamics of Liquid Helium II. Phys. Rev. B, Vol. 48, 6276-6283.

Mongiovì, M.S. (2001). Extended irreversible thermodynamics of liquid helium II: boundary condition and propagation of fourth sound. Physica A, Vol. 291, 518-537.

Mongiovì, M.S. \& Jou, D. (2005). Superfluid turbulence in rotating containers: phenomenological description of the influence of the wall. Phys. Rev. B, Vol. 72, 104515 (8 pages).

Mongiovì, M.S. \& Jou, D. (2007). Thermodynamical derivation of a hydrodynamical model of inhomogeneous superfluid turbulence. Phys. Rev. B, Vol. 75, 024507 (14 pages).

Müller, I. \& Ruggeri, T. (1998). Rational Extended Thermodynamics, Springer-Verlag, New York.

Nemirovskii, S.K. \& Lebedev, V.V.(1983). The hydrodynamics of superfluid turbulence. Sov. Phys. JETP, Vol. 57, 1009-1016.

Nemirovskii, S.K. \& Fiszdon, W.(1995). Chaotic quantized vortices and hydrodynamic processes in superfluid helium. Reviews of Modern Physics, Vol. 67, 37-84.

Onsager, L.(1949). Statistical hydrodynamics. Nuovo Cimento, Vol. 6, 249-250.

Osborne, D.V.(1950). The Rotation of Liquid Helium II. Proc. Phys. Soc. A, Vol. 63, 909-912.

Peruzza \& Sciacca, (2007). Waves propagation in turbulent superfluid helium in presence of combined rotation and counterflow. Physica B, Vol. 398, 8-17.

Schwarz, K.W.(1982). Generating superfluid turbulence from simple dynamical rules. Phys. Rev. Lett., Vol. 49, 283-285.

Schwarz, K.W.(1985). Three-dimensional vortex dynamics in superfluid ${ }^{4} \mathrm{He}$, I. Line-line and line boundary interactions. Phys. Rev. B, Vol. 31, 5782-5804.

Schwarz, K.W.(1988). Three-dimensional vortex dynamics in superfluid ${ }^{4}$ He. Phys. Rev. B, Vol. 
38, 2398-2417.

Sciacca, M., Mongiovì, M.S. \& Jou, D. (2008). A mathematical model of counterflow superfluid turbulence describing heat waves and vortex-density waves. Math. Comp. Mod., Vol. 48, 206-221.

Snyder, H.A., Putney, Z. (1966). Angular dependence of mutual friction in rotating He II. Phys. Rev., Vol. 150, 110-117.

Sonin, E.B. (1987). Vortex oscillations and hydrodynamics of rotating superfluids. Rev. Mod. Phys., Vol. 59, 87-155.

Swanson, C.E., Barenghi, C.F.\& Donnelly, R.J.(1983). Rotation of a tangle of quantized vortex lines in He II. Phys. Rev. Lett., Vol. 50, 190-193.

Tisza, L. (1938). Transport phenomena in He II. Nature, Vol. 141, 913.

Tough, J.T., (1982). Superfluid turbulence, In: Progress of Low Temperature Physics Vol. III, Brewer, D.F., (Ed.), 133, North-Holland, Amsterdam.

Tsubota, M., Araki, T. \& Barenghi, C.F.(2003). Rotating superfluid turbulence. Phys. Rev. Lett., Vol. 90, 205301 (4 pages).

Tsubota, M., Araki, T. \& Vinen, W.F.(2003). Diffusion of an inhomogeneous vortex tangle. Physica B, Vol. 329-333, 224-225.

Tsubota, M., Araki, T. \& Barenghi, C.F.(2004). Vortex Tangle Polarized by Rotation. Jour. Low Temp. Phys., Vol. 134, 471-476.

Tsubota, M., Barenghi, C.F., Araki, T. \& Mitani, A. (2004). Instability of vortex array and transitions to turbulence in rotating helium II. Phys. Rev. B, Vol. 69, 134515 (12 pages).

van Beelen, H., van Joolingen, W. \& Yamada, K. (1988). On a balance equation for superfluid vorticity in capillary flow of helium II. Physica B, Vol. 153, 248-253.

Vinen, W.F. (1957). Mutual friction in a heat current in liquid helium II. I. Experiments on steady heat current. Proc. Roy. Soc., Vol. A240, 114-127.

Vinen, W.F. (1957). Mutual friction in a heat current in liquid helium II. II. Experiments on transient effects. Proc. Roy. Soc., Vol. A242, 128-143.

Vinen, W.F. (1957). Mutual friction in a heat current in liquid helium II. III. Theory of the mutual friction. Proc. Roy. Soc., Vol. A240, 493-515.

Vinen, W.F. (1958). Mutual friction in a heat current in liquid helium II. IV. Critical heat current in wide channels. Proc. Roy. Soc., Vol. A243, 400-413.

Vinen, W.F. (2000). Classical character of turbulence in quantum fluid. Phys. Rev. B, Vol. 61, 1410-1420.

Vinen \& Niemela, (2002). Quantum Turbulence. Jour. Low Temp. Phys., Vol. 128, 167-231.

Whitham, J. (1974). Linear and Nonlinear Waves, Wiley, New York.

Yamada, K., Kashiwamura, S.\& Miyake, K. (1989). Stochastic theory of vortex tangle in superfluid turbulence. Physica B, Vol. 154, 318-326. 


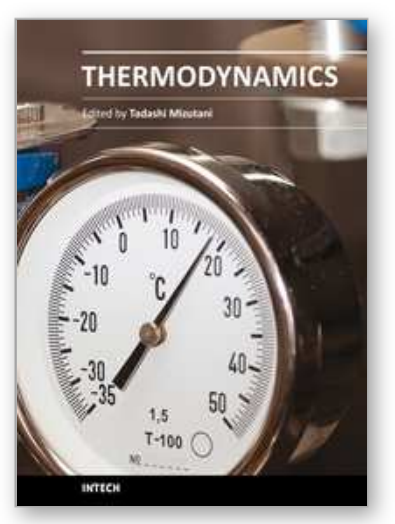

\author{
Thermodynamics \\ Edited by Prof. Mizutani Tadashi
}

ISBN 978-953-307-544-0

Hard cover, 440 pages

Publisher InTech

Published online 14, January, 2011

Published in print edition January, 2011

Progress of thermodynamics has been stimulated by the findings of a variety of fields of science and technology. The principles of thermodynamics are so general that the application is widespread to such fields as solid state physics, chemistry, biology, astronomical science, materials science, and chemical engineering. The contents of this book should be of help to many scientists and engineers.

\title{
How to reference
}

In order to correctly reference this scholarly work, feel free to copy and paste the following:

D. Jou, M.S. Mongiovì, M. Sciacca, L. Ardizzone and G. Gaeta (2011). Hydrodynamical Models of Superfluid Turbulence, Thermodynamics, Prof. Mizutani Tadashi (Ed.), ISBN: 978-953-307-544-0, InTech, Available from: http://www.intechopen.com/books/thermodynamics/hydrodynamical-models-of-superfluid-turbulence

\section{INTECH}

open science | open minds

\section{InTech Europe}

University Campus STeP Ri

Slavka Krautzeka 83/A

51000 Rijeka, Croatia

Phone: +385 (51) 770447

Fax: +385 (51) 686166

www.intechopen.com

\section{InTech China}

Unit 405, Office Block, Hotel Equatorial Shanghai

No.65, Yan An Road (West), Shanghai, 200040, China

中国上海市延安西路65号上海国际贵都大饭店办公楼405单元

Phone: +86-21-62489820

Fax: $+86-21-62489821$ 
(C) 2011 The Author(s). Licensee IntechOpen. This chapter is distributed under the terms of the Creative Commons Attribution-NonCommercialShareAlike-3.0 License, which permits use, distribution and reproduction for non-commercial purposes, provided the original is properly cited and derivative works building on this content are distributed under the same license. 